\title{
Reduced Specificity of Autobiographical Memory and Depression: The Role of Executive Control
}

\author{
Tim Dalgleish \\ Medical Research Council Cognition and Brain Sciences Unit
}

Ann-Marie J. Golden

Medical Research Council Cognition and Brain Sciences Unit

\author{
J. Mark G. Williams \\ University of Oxford
}

Nicola Perkins

University of Bath

Phillip J. Barnard

Medical Research Council Cognition and Brain Sciences Unit

Rachael Elward

University of Cardiff

Edward Watkins

University of Exeter

\begin{abstract}
It has been widely established that depressed mood states and clinical depression, as well as a range of other psychiatric disorders, are associated with a relative difficulty in accessing specific autobiographical information in response to emotion-related cue words on an Autobiographical Memory Test (AMT; J. M. G. Williams \& K. Broadbent, 1986). In 8 studies the authors examined the extent to which this relationship is a function of impaired executive control associated with these mood states and clinical disorders. Studies 1-4 demonstrated that performance on the AMT is associated with performance on measures of executive control, independent of depressed mood. Furthermore, Study 1 showed that executive control (as measured by verbal fluency) mediated the relationship between both depressed mood and a clinical diagnosis of eating disorder and AMT performance. Using a stratified sample in Study 5, the authors confirmed the positive association between depressed mood and impaired performance on the AMT. Studies 6-8 involved experimental manipulations of the parameters of the AMT designed to further indicate that reduced executive control is to a significant extent driving the relationship between depressed mood and AMT performance. The potential role of executive control in accounting for other aspects of the AMT literature is discussed.
\end{abstract}

Keywords: depression, executive control, autobiographical memory, overgeneral memory, working memory capacity

When asked to recall specific autobiographical memories of events with a duration of less than 1 day in response to word cues, people sometimes produce overly general responses. For example, the cue "vacation" might prompt the generic recollection "I enjoyed all of my vacations as a child" instead of the required specific response "I remember the day that we went to Disneyland last year." Williams and Broadbent (1986) serendipitously discovered that this difficulty in producing specific memories was more widespread in their group of depressed parasuicide patients than in a matched control sample. Since this initial study, reduced auto-
Tim Dalgleish, Ann-Marie J. Golden, Phillip J. Barnard, Emotion Research Group, Medical Research Council Cognition and Brain Sciences Unit, Cambridge, England; J. Mark G. Williams, Department of Psychiatry, University of Oxford, Oxford, England; Nicola Perkins, Cecilia Au Yeung, and Victoria Murphy, Department of Psychology, University of Bath, Bath, England; Lisa Feldman Barrett, Department of Psychology, Boston College; Rachael Elward, Department of Psychology, University of Cardiff, Cardiff, Wales; Kate Tchanturia, Eating Disorders Unit, Department of Psychiatry, Institute of Psychiatry, Kings College, University of London, London, England; Edward Watkins, Department of Psychology, University of Exeter, Exeter, England.
Tim Dalgleish, Nicola Perkins, Ann-Marie J. Golden, Phillip J. Barnard, Cecilia Au Yeung, Victoria Murphy, and Rachael Elward were supported by the Medical Research Council. J. Mark G. Williams and Kate Tchanturia were supported by grants from the Wellcome Trust. Our thanks go to John Duncan, Andrew Mathews, Andrew Lawrence, and Lyndsey Drummond for helpful discussions about this work.

Correspondence concerning this article should be addressed to Tim Dalgleish, Emotion Research Group, Medical Research Council Cognition and Brain Sciences Unit, 15 Chaucer Road, Cambridge, England CB2 2EF E-mail: tim.dalgleish@mrc-cbu.cam.ac.uk 
biographical memory specificity (AMS) has been found to be a characteristic of performance on this particular task (the Autobiographical Memory Test [AMT]; Williams \& Broadbent, 1986) in a number of clinical groups. These include individuals suffering from clinical depression (e.g., Brewin, Reynolds, \& Tata, 1999; Brittlebank, Scott, Williams, \& Ferrier, 1993; Kuyken \& Dalgleish, 1995; Wessel, Meeren, Peeters, Arntz, \& Merckelbach, 2001), posttraumatic stress disorder (PTSD; e.g., McNally, Litz, Prassas, Shin, \& Weathers, 1994), acute stress disorder (e.g., Harvey, Bryant, \& Dang, 1998), eating disorders (e.g., Dalgleish et al., 2003), and borderline personality disorder (e.g., Startup et al., 2001). Furthermore, reduced AMS has been found to be associated with levels of subclinical depressed mood in both naturalistic (e.g., Ramponi, Barnard, \& Nimmo-Smith, 2004) and mood-induction studies (e.g., Au Yeung, Dalgleish, Golden, \& Schartau, 2006).

A number of key findings have suggested that reduced AMS is more than simply a cognitive curiosity. Instead, the data indicate that reduced AMS indexes one or more fundamental cognitive processes closely linked to the onset, maintenance, and recovery from a variety of clinical and subclinical states.

The first such key finding was initially reported by Brittlebank et al. (1993), who showed that reduced AMS predicted later clinical recovery in individuals with major depressive disorder, over and above initial levels of depressive symptoms. This longitudinal effect has been replicated in other depressed samples (e.g., Dalgleish, Spinks, Yiend, \& Kuyken, 2001; Hipwell, Reynolds, \& Pitts Crick, 2004; Mackinger, Loschin, \& Leibetseder, 2000; but see Brewin et al., 1999). Similarly, Harvey et al. (1998) found that reduced AMS posttrauma in motor vehicle accident survivors predicted higher later levels of posttraumatic distress (but see Kangas, Henry, \& Bryant, 2005).

The second key finding is that participants in Brittlebank et al.'s (1993) study who recovered from depression still showed reduced AMS relative to control participants, indicating that reduced AMS is a stable marker in recovered or remitted clinical groups and not just a function of acute depressive symptomatology. This was demonstrated more systematically by Mackinger, Pachinger, Leibetseder, and Fartacek (2000), who compared never-depressed and recovered-depressed women on the AMT and found relatively reduced AMS in the recovered sample.

Finally, reduced AMS has been shown to relate to impoverished problem-solving ability, with participants low in specificity finding it difficult to generate solutions on social problem-solving tasks (e.g., Goddard, Dritschel, \& Burton, 1996). This fact, along with the related finding that reduced AMS is associated with a difficulty in generating specific simulations of the future (Williams, Ellis, Tyers, MacLeod, \& Rose, 1996), suggests that reduced AMS is of potential clinical significance when considering impairments in day-to-day cognitive functioning in some patient groups.

Taken together these key findings suggest that a better understanding of the psychological processes involved in reducing AMS will yield insight into cognitive factors implicated in the onset and maintenance of depressed mood, clinical depression, and other psychopathological states as well as day-to-day cognitive impairments associated with a range of clinical disorders.

Theoretical attempts to understand the source of reduced AMS in the affective science and abnormal psychology literature have been strongly influenced by another aspect of the AMS data-the relationship between reduced AMS and a history of trauma. The prototypical study in this area was by Kuyken and Brewin (1995), who examined AMT performance in depressed women with and without a reported history of childhood abuse. Women with a history of abuse showed reduced AMS relative to those women who had not been abused. Furthermore, increased levels of current intrusion and avoidance symptoms concerning the abuse correlated positively with the reduction in AMS. Since this initial study, this relationship between reduced AMS and both a history of trauma and greater intrusive and avoidant symptomatology following trauma has been replicated several times and for various kinds of traumatic experience (e.g., Dalgleish et al., 2003; de Decker, Hermans, Raes, \& Eelen, 2003; Henderson, Hargreaves, Gregory, \& Williams, 2002; though see Kuyken, Howell, \& Dalgleish, 2006).

Building on these findings, the most consensual theoretical explanation of reduced AMS to date has been the affect regulation hypothesis (Williams, Stiles, \& Shapiro, 1999). The affect regulation hypothesis accounts for reduced AMS in terms of difficulties in searching a self-memory system (M. A. Conway \& Pleydell-Pearce, 2000). The proposal is that to recall a specific autobiographical memory to a cue word, one's first step is to generate a restricted set of categorical descriptors constrained by the cue word. These descriptors are then used to search the memory system for memories that relate to that cue word. Subsequent sets of descriptors are then generated iteratively, allowing an increasingly refined search of this categorical subset of memories in order to retrieve specific episodes that comply with the task instructions. Williams, Stiles, and Shapiro (1999) proposed that to progress beyond the categorical descriptor stage during memory search to a more refined interrogation of the specific memory database, one must inhibit unneeded categorical descriptors in some way. ${ }^{1}$ Failure to inhibit these descriptors, it is proposed, will lead to the generation of overly general responses (reduced AMS) to cue words.

Williams has further suggested that children learn to inhibit irrelevant descriptors during memory search as a function of normal development but that trauma in childhood impairs this learning (Williams et al., in press). The heart of the affect regulation hypothesis is that painful emotional information is avoided by

\footnotetext{
${ }^{1}$ The label inhibition is used here and throughout the rest of the article as a term of convenience. Within cognitive psychology a distinction has been drawn between concepts of strong and weak inhibition (e.g., Dalgleish, Mathews, \& Wood, 1999). Strong inhibition refers to putative situations in which processes or representations are directly inhibited within the cognitive system. The notion of strong inhibition is somewhat controversial, with many arguing that there is no need for such a construct at the functional level of explanation (see MacLeod, Dodd, Sheard, Wilson, $\&$ Bibi, 2003, for a provocative discussion). In contrast, weak inhibition refers to situations in which a given process or representation is less activated than competing representations, as if it has been inhibited. However, weak inhibition can potentially arise entirely as a function of the competing representations or processes being selectively activated; in other words, weak inhibition does not require that strong inhibition is taking place, though of course it does not preclude it either. For the present purposes, the term inhibition is used in this weak sense, whereby it is as yet unclear whether strong inhibitory mechanisms are at work (cf. Dagenbach \& Carr, 1994).
} 
aborting memory search processes at a nonspecific level of analysis. Consequently, the argument goes, individuals with a history of childhood trauma will be less able to inhibit categorical descriptors during memory search and will therefore produce relatively reduced AMS on the AMT. As childhood trauma is a key correlate of depression and other clinical states (e.g., Boudewyn \& Liem, 1995), the affect regulation hypothesis provides a plausible account of reduced AMS in a range of clinical conditions.

Although the affect regulation hypothesis has clear face validity as an account of reduced AMS, it seems unlikely to be a complete account. For example, it is difficult to see how the affect regulation hypothesis can offer a plausible conceptualization of reduced AMS following a negative mood induction in healthy individuals with no history of depression (Au Yeung et al., 2006) or in healthy individuals following category generation on related themes (Barnard, Watkins, \& Ramponi, 2006). An additional or possibly alternative explanation for reduced AMS, and one that has the benefit of parsimony in that it is consistent with the wider theoretical literature on depression (and indeed other forms of emotional disorder), is that the phenomenon results from relatively poor executive or attentional control on the part of depressed individuals in the face of a cognitively demanding task-in this case the AMT.

Although there is limited consensus about exactly which cognitive operations fall under the rubric of executive control, let alone about which processes might underpin executive control within a psychological system (see Miyake \& Shah, 1999, Question 2), it is generally agreed that executive control broadly encapsulates the set of cognitive processes that are responsible for the planning, initiation, sequencing, and monitoring of complex goal-directed behavior in the face of distracting information. Relatively impaired executive control in depression is a key empirical finding in the literature (see Burt, Zembar, \& Niederehe, 1995; Hartlage, Alloy, Vazquez, \& Dykman, 1993, for reviews) and a prominent feature of a number of influential theoretical models of the disorder (Barrett, Tugade, \& Engle, 2004; Ellis \& Ashbrook, 1988; Hasher \& Zacks, 1979; Hertel \& Rude, 1991). ${ }^{2}$

A prototypical model of impaired executive control in depression has been articulated by Hertel and colleagues (e.g., Hertel \& Hardin, 1990; Hertel \& Rude, 1991; see Hertel, 2000, for an overview) in their cognitive-initiative account. The central thesis of the cognitive-initiative approach is that where executive control processes are poorly constrained by a given task (for example the AMT), depressed individuals should exhibit impaired performance due to a deficit in the ability to initiate relevant strategies or generate appropriate hypotheses when performing the task. Within this type of executive control framework, reduced AMS on the AMT may be a consequence of impoverished retrieval strategies during memory search in certain individuals or groups, a difficulty in maintaining or applying the task instructions to be specific during the task itself, and/or problems with inhibiting inappropriate (i.e., overgeneral) candidate memory responses on the task.

It is interesting to note that in the same essay in which Williams (1996) proposed the affect regulation hypothesis he also highlighted a putative role for executive processes in defining the reduced AMS effect. It was assumed that once memory search had been aborted, further iterations of the retrieval process would give rise to the activation of other intermediate descriptions. After a number of such iterations a more highly elaborated network of categoric memories would exist so that, under conditions in which executive control was compromised, such information would be more likely to be generated as responses on future memory searches (Williams, 1996).

Despite these predictions, a clear empirical link between reduced AMS and executive control has yet to be established. The results from the handful of studies relevant to this issue have generally been negative, mixed, or inconclusive. For example, Williams and Broadbent (1986) found that reduced AMS was not associated with category fluency (for vegetables or boys' names) or processing speed (the time taken to judge a series of 50 "silly sentences" as true or false; Baddeley, Emslie, \& Nimmo-Smith, 1992). Other studies found a negative association between performance in a word fluency task and reduced AMS (e.g., Williams \& Dritschel, 1992). Still others indicated some role for general cognitive ability (including executive control processes) in AMT performance but have suggested that such processes offer a farfrom-complete explanation of the reduced AMS effect. For example, Park, Goodyer, and Teasdale (2002) matched two samples of adolescents for IQ but still found that individuals in the depressed group gave significantly fewer specific responses. Similarly, Wessel et al. (2001) found that educational level contributed significantly to the prediction of AMS but that between-group differences remained after controlling for this variable.

One reason for the equivocal nature of the findings to date may be that the research seeking to account for the reduced AMS effect has not focused clearly enough or exclusively on tasks that load heavily on executive control. In fact, depression (and indeed other forms of psychopathology) does not seem to be associated with impairments on all forms of cognitive task but rather seems to relate specifically to tasks in which effective executive control is particularly important (e.g., Hartlage et al., 1993). The rationale for the present series of studies was therefore to investigate systematically for the first time the extent to which reduced AMS on the AMT is an example of poor task performance on a paradigm that loads significantly on executive control and that is associated with depressed mood and/or various clinical states.

To this end, in the first study we sought to examine, through reanalysis of existing data in patients with eating disorders, the relationship between AMT performance and verbal fluency-a classic executive control measure (Rosen \& Engle, 1997). We also included a mediational analysis in Study 1 in order to investigate whether the relationship between both an eating disorder diagnosis and depressed mood, on the one hand, and reduced AMS, on the other, was significantly accounted for by verbal fluency scores.

The aim behind Studies 2-4 was to further establish a nomological net (Cronbach \& Meehl, 1955) for the AMT by demonstrating a clear relationship (independent of depression) between AMT performance and performance on other nonautobiographical and generally emotion-unrelated tasks that themselves depend on executive control processes. In these three studies we therefore exploited naturally occurring individual differences in executive control capabilities in unselected samples to establish the existence

\footnotetext{
${ }^{2}$ Not all of these theoretical models talk explicitly about executive control. For example, Ellis's resource allocation model (e.g., Ellis \& Ashbrook, 1988) uses a spatial metaphor whereby impaired task performance in depression is a function of a reduction in executive "resources."
} 
of such a relationship, and in each case depressed mood was included as a covariate in the key analyses.

Having established that the AMT is a task associated with other executive control measures independent of depression (Studies 1-4) and that executive control mediates the relationship between depression (as well as eating disorder diagnosis) and reduced AMS in a clinical sample, in Studies 5-8 we sought to further show that the diminished executive control routinely associated with depressed mood (e.g., Hartlage et al., 1993) is an important factor in determining the patterns of AMT performance in depression. To do this we first replicated the significant association between depressed mood and AMT performance in a stratified dysphoric sample, using the standard version of the AMT from the extant literature (Study 5). We then manipulated the task parameters of this standard AMT in ways that would interact with individual differences in executive control (such as those that occur naturally in correspondence with individual differences in levels of depressed mood; Studies 6-8). In these latter four studies, levels of depressed mood were not included as a covariate, because the nature and direction of the relationship between depression and AMS, in the face of variations of the AMT parameters, were the key variables of interest.

\section{Study 1: AMS and Verbal Fluency in Patients With an Eating Disorder and Matched Control Participants-A Reanalysis of Existing Data}

In a previous study, Dalgleish et al. (2003) described how patients with a diagnosis of an eating disorder exhibited reduced AMS on the AMT relative to healthy control participants. Furthermore, these results indicated that reduced AMS, indexed by numbers of overgeneral memories, was associated with level of selfreported parental abuse (mirroring earlier data; e.g., Kuyken \& Brewin, 1995). A proportion of the participants in Dalgleish et al.'s (2003) study also took part in a separate study (Godley, Tchanturia, MacLeod, \& Schmidt, 2001) in which participants had to complete a standard verbal fluency test-generating as many words beginning with the letter $s$ as they could in $1 \mathrm{~min}$. Verbal fluency is regarded as a broad measure of executive control (e.g., Rosen \& Engle, 1997). This allowed us in this reanalysis of the data to examine the degree to which verbal fluency was associated with reduced AMS on the AMT. We also wanted to examine whether substituting participants' verbal fluency scores in place of their AMS scores in the analyses previously reported (Dalgleish et al., 2003) generated a similar pattern of findings. Participants in Godley et al.'s (2001) study completed a test in which they generated as many specific putative future autobiographical experiences as they could in relation to different future time domains (the Future Fluency Test). This allowed us to examine whether reduced AMS in our healthy control participants was related to a reduced ability to generate specific events about the future on the Future Fluency Test (similar to that found by Williams et al., 1996; see the present article's introduction), again with a view to examining subsequently whether this relationship would be the same if we substituted AMS scores with verbal fluency scores in the same control participants. For all of these planned analyses, we covaried scores on a measure of depression to ensure that any relationships that emerged were not simply due to both variables being proxy depression measures.
Finally, we examined whether verbal fluency scores mediated the relationships between depression or eating disorder diagnosis (as the predictors) and AMS (as the criterion), to establish whether the relationship between depression or eating disorder and AMS could be significantly accounted for by variations in executive control.

Our specific hypotheses for Study 1 were therefore as follows:

Hypothesis 1: There will be a significant positive correlation between AMS and verbal fluency across all participants, with self-reported depression levels partialed out.

Hypothesis 2: Participants in the eating disorder group should score lower on the verbal fluency test compared with control participants.

Hypothesis 3: Verbal fluency performance in the eating disorder group should correlate negatively with degree of selfreported parental abuse, with levels of self-reported depression partialed out.

Hypothesis 4: AMS should be significantly positively correlated with numbers of specific events generated on the Future Fluency Test in the healthy control participants.

Hypothesis 5: This relationship should be mirrored by that between verbal fluency and performance on the Future Fluency Test.

Hypothesis 6: Verbal fluency will significantly mediate the relationships between depression and AMS and between eating disorder diagnosis and AMS.

\section{Method}

\section{Participants}

The clinical participants were 32 patients with a primary diagnosis of eating disorder. ${ }^{3}$ Twenty were diagnosed with anorexia nervosa (restricting subtype, $n=15$; binge-purge subtype, $n=5$ ), and 12 were diagnosed with bulimia nervosa according to the Diagnostic and Statistical Manual of Mental Disorders (4th ed.; DSM-IV; American Psychiatric Association, 1994) criteria.

The healthy control group was composed of 19 participants recruited through an established participant pool at the Institute of Psychiatry, London. Control participants had no self-reported history of psychiatric or eating problems and were comparable with the clinical participants on age, education level, and gender ratio (see the Results and Discussion section). Further details about all participants and their selection and diagnosis can be found in Dalgleish et al.'s (2003) article.

\section{Materials and Measures}

The AMT. The AMT was exactly as described by Kuyken and Dalgleish (1995). Ten emotional words (from Williams \& Broadbent, 1986) were used to cue the memories: five pleasant (happy, safe, interested, successful, and surprised) and five unpleasant (sorry, angry, clumsy, hurt, and lonely). Participants were given $1 \mathrm{~min}$ in each case to retrieve a specific

\footnotetext{
3 This sample size is lower than that reported in Dalgleish et al.'s (2003) study because a number of the eating disordered patients did not complete the verbal fluency task.
} 
autobiographical memory - a specific time and place when something happened to them. Participants were told that the memory they recalled could be something that happened recently or a long time ago and that it could be an important or trivial event but that the memory should be of something that happened at a particular time on a particular day. Examples of acceptable and unacceptable responses were given. A printed version of the recall instructions was given to the participants to read. Cue words were presented on $12.5 \mathrm{~cm} \times 7.5 \mathrm{~cm}$ laminated cards and were written in black ink in capital letters $3.5 \mathrm{~cm}$ high. Words were presented in a separate random order for each participant. To ensure that participants understood the instructions, we gave two practice cues (relieved and tired).

Generated memories were tape-recorded and transcribed for coding according to the criteria laid down by Williams (1992). Specific memories were defined as events that happened in a particular instance or lasted for 1 day or less. Nonspecific memories included extended memories (events that lasted for longer periods of time) and categoric memories (events that occurred repeatedly over a period of time). If the participants failed to recall a memory within the time limit or talked about things that were not memories (e.g., an opinion that is associated with the cue), their responses were classed as "no memories." If the type of memory that the participants recalled was unclear, or if participants retrieved the same memory to more than one cue or offered responses that related to future events, they were prompted with the words "What is the memory that you are thinking of there?" or "Can you tell me a bit more about that memory?" For the studies reported in this article, all analyses focus on numbers of specific memories; in other words, AMS scores refer to number of specific memories generated to the entire set of words. Interrater agreement between Ann-Marie J. Golden and Tim Dalgleish on 50\% of the retrieved memories for Study $1(n=305)$ indicated good reliability $(\kappa=.78)$ comparable with previous studies. The same raters were used for all subsequent studies.

Thurstone Verbal Fluency Test. A standard version of the verbal fluency test from Lezak (1995) was used. Participants were asked to list as many words as they could in $1 \mathrm{~min}$ beginning with the letter $s$. Participants were informed that repetitions, proper nouns (e.g., Switzerland), and more than one word of the same origin (e.g., swim, swimming) were not acceptable answers, and they were given examples of each. The total number of acceptable words produced was the verbal fluency score.

Early experience. Perception of early family experience was assessed using the Measure of Parenting Style (Parker et al., 1997). This is a 15-item self-report measure with scales assessing parental overcontrol, indifference, and abuse. The Abuse subscale is the focus of the present analyses. For more details see Dalgleish et al.'s (2003) study.

Future Fluency Test. This was as described in Godley et al.'s (2001) study. Participants were asked to generate as many specific future events as they could for three different time periods-the next week, including the current day; the next year; and the next 5-10 years. Participants were asked to generate positive and negative events separately. There was a time limit of $1 \mathrm{~min}$ for each period-valence combination. For the purposes of the present study a composite score of the total number of events generated across the six conditions was used.

\section{Procedure}

In this study and in all of the studies reported here, participants were tested individually and face-to-face in a quiet testing environment, and all testing was carried out by the same experimenter. Participants also completed the Hospital Anxiety and Depression Scale (HADS; Zigmond \& Snaith, 1983), a self-report measure of experienced anxiety and depression, immediately prior to the AMT.

\section{Results and Discussion}

Descriptive statistics for the key variables across the two groups-eating disordered participants and control participantsare presented in Table 1. For the present study and all subsequent studies in this article, analyses examining a priori hypotheses are one-tailed. All other analyses are two-tailed. Where data do not meet the criteria for parametric statistics this is noted in the text and suitable transformations and/or nonparametric methods are applied.

Our first hypothesis of a significant relationship between AMS and verbal fluency across the whole sample, after partialing out self-reported depression on the HADS - which correlated significantly with AMS, $r(49)=-.35, p<.05$-was supported with poorer verbal fluency associated with reduced AMS, $\operatorname{pr}(48)=.47$, $p<.01$. Furthermore, in a regression analysis with both HADSDepression scores and verbal fluency as predictors (accounting for $28 \%$ of the variance in AMS), only verbal fluency accounted for significant unique variance $(18 \%), t(48)=3.55, p<.001$, versus $4 \%$ for HADS-Depression scores.

The critical analyses involving AMS were then repeated using verbal fluency scores in place of AMS scores to examine Hypotheses 2 and 3. The results indicated that verbal fluency scores, as predicted, differed significantly across groups, $t(49)=2.96, p<$ .01 , with eating disordered participants showing poorer fluency.

Table 1

Data for the Demographic, Memory, Future Fluency, Verbal Fluency, Self-Reported Abuse, and Mood Variables in Study 1

\begin{tabular}{|c|c|c|c|c|}
\hline \multirow[b]{2}{*}{ Variable } & \multicolumn{2}{|c|}{$\mathrm{ED}^{\mathrm{a}}$} & \multicolumn{2}{|c|}{ Control participants ${ }^{\mathrm{b}}$} \\
\hline & $M$ & $S D$ & $M$ & $S D$ \\
\hline Age (years) & 26.78 & 8.10 & 27.73 & 5.96 \\
\hline HADS-Depression & 11.38 & 4.99 & 2.00 & 1.89 \\
\hline Verbal fluency & 16.47 & 4.06 & 20.32 & 5.15 \\
\hline MOPS-Abuse ${ }^{\mathrm{c}}$ & 6.96 & 5.87 & 2.05 & 2.75 \\
\hline No. specific memories on the AMT & 6.03 & 2.43 & 8.63 & 1.38 \\
\hline Future Fluency Test ${ }^{\mathrm{d}}$ & 24.43 & 9.99 & 27.00 & 6.23 \\
\hline
\end{tabular}

Note. $\quad$ ED $=$ eating disordered participants; HADS-Depression $=$ Hospital Anxiety and Depression ScaleDepression subscale score; MOPS-Abuse = Measure of Parenting Style-Abuse subscale score, summed across mother and father; AMT $=$ Autobiographical Memory Test.

${ }^{\mathrm{a}} n=32$ (3 men, 29 women). ${ }^{\mathrm{b}} n=19$ (1 man, 18 women). ${ }^{\mathrm{c}}$ Four of the ED group did not complete this measure. ${ }^{\mathrm{d}}$ Two of the ED group and 1 control participant did not complete this measure. 
However, in the eating disorder group, the correlation between fluency scores and Measure of Parenting Style-Abuse subscale scores did not approach significance, $\operatorname{pr}(30)=.02, n s$, thus providing no support for Hypothesis 3.

The relationship between AMS and the number of future events generated in the healthy participants on the Future Fluency Test (Hypothesis 4) was significant and positive, $r(16)=.66, p<.01$, and remained once depression - which correlated with Future Fluency Scores, $r(16)=.49, p<.05$-was partialed out, $\operatorname{pr}(15)=$ $.57, p<.01$, consistent with the findings reported by Williams et al. (1996) for the specificity of future imagined events.

We next established that in the healthy participants, verbal fluency scores were significantly positively correlated with the total number of emotional future events generated on the Future Fluency Test, $r(16)=.46, p<.03$. This remained significant when self-reported depression was partialed out, $\operatorname{pr}(15)=.44, p<$ .05 , in line with Hypothesis 5.

Finally, we conducted two sets of mediational analyses to examine, first, whether the relationship between diagnostic status (eating disorder vs. control participants) and AMS was mediated by verbal fluency scores and, second, whether the relationship between depressed mood (on the HADS-Depression subscale) across all participants and AMS was mediated by verbal fluency scores. These analyses were conducted using the bootstrapping procedures recommended for smaller samples (MacKinnon, Lockwood, Hoffman, West, \& Sheets, 2002; Preacher \& Hayes, 2004) and operationalized in an SPSS macro by Preacher and Hayes (2004). In our analyses we used 5,000 bootstrap resamples of the data with replacement. Statistical significance with alpha at .05 is indicated by the $95 \%$ confidence intervals not crossing zero. The results revealed a significant mediation effect of verbal fluency with respect to the relationship between eating disorder diagnosis and AMS, Sobel statistic $=.75, S E=.36,95 \%$ confidence intervals $=0.15,1.56$, and between HADS-Depression and AMS, Sobel statistic $=-.05, S E=.03,95 \%$ confidence intervals $=$ $-.12,-.002$.

Taken together, these data provide evidence for significant shared variance between AMS performance and a standardized measure of executive control (verbal fluency; Rosen \& Engle, 1997) that, on face value, has little or nothing to do with autobiography or emotion. Crucially, this significant shared variance is independent of depression. It is interesting to note that the data also indicate that the relationship between AMS and self-reported abuse in the clinical group was somewhat independent of executive control, at least as indexed by verbal fluency scores. This issue is revisited in the General Discussion. Finally, the mediational analyses suggest that significant aspects of the relationships between both eating disorder diagnosis and depressed mood, on the one hand, and AMS, on the other, can be accounted for by variations in executive control.

\section{Study 2: The Relationship Between AMS and Scores on a Range of Tests of Executive Control}

In Study 2 we again explored the relationship between AMS and executive control, independent of self-reported depression: this time in an unselected sample of participants. We used a wide range of tasks that are accepted as measures of executive control in the neuropsychological literature in order to permit a broad examina- tion of our hypothesis that a significant amount of the variance in AMS would be accounted for by executive control measures that have little or nothing explicitly to do with autobiography or emotion.

\section{Method}

\section{Participants}

To determine sample size, we conducted a power analysis based on the large correlation (Cohen, 1988) between verbal fluency and AMS from Study 1. To obtain $80 \%$ power with an alpha of .05 that a similar-sized correlation would be different from zero in a given direction in the present study, a sample size of 24 was indicated (Cohen, 1988). This was used as a guide for the present study and subsequent studies unless otherwise indicated. On this basis, 24 unselected participants (5 men, 19 women) were recruited from the Cognition and Brain Sciences Unit (CBU) volunteer panel. ${ }^{4}$ The mean age of participants was 40.83 years $(S D=9.18$ years).

\section{Materials and Measures}

The AMT. The AMT was as for Study 1, with the exception that a greater number of cue words were used, to provide a wider range of scores to facilitate correlational analyses. In this study, 12 positive and 12 negative cue words were selected (Brittlebank et al., 1993), balanced for ratings of emotion-relatedness (Brittlebank et al., 1993) and frequency (Kučera \& Francis, 1967). Participants practiced with three neutral words (gigantic, grass, and absence).

Thurstone Verbal Fluency Test. The version of this test from Study 1 was used (Lezak, 1995).

Design Fluency Test. A nonverbal equivalent of the verbal fluency test, the design fluency test (Jones-Gotman \& Milner, 1977), was used to assess divergent thinking. Participants were asked to "invent" as many drawings - which could not represent actual objects or shapes-in 1 min. Participants were told to avoid simply drawing scribbles, spending too long on any one drawing, or inventing two similar looking drawings. Practice was given in which participants invented three drawings and were given feedback with reference to the instructions of the task. The total number of drawings, the number of errors (i.e., highly similar looking drawings, drawings of actual objects, etc.), and the corrected score were recorded.

Alternate Uses Test. A short version of this test as described by Lezak (1995) was used as a further measure of divergent thinking. Participants were asked to generate as many creative uses for a brick as they could in $1 \mathrm{~min}$. Participants were instructed to avoid uses that were typical (e.g., building a wall) or nonsensical (e.g., wearing as a hat). Examples of unacceptable answers were provided. The total number of appropriate uses and the number of errors (i.e., typical or nonsensical uses) were recorded.

Wechsler Adult Intelligence Scale-III (WAIS-III; Wechsler, 1998) Block Design. Participants were administered the Block Design subtest as set out by the WAIS-III manual (Wechsler, 1998). Participants had to arrange a number of blocks with sides of different colors in a configuration that matched either the experimenter's blocks or a printed design. There was a time limit on each design. A total raw score was converted to an age-related scaled score (Wechsler, 1998) for each participant.

Porteus Maze Test. The Vineland Revision set of Porteus Maze Tests was administered (Porteus, 1965) as a measure of planning. Tests Year VII to Adult I were used. Participants were presented with a series of mazes and were asked to draw a line, from the beginning point, out of the maze. Participants were asked to complete each maze as quickly as possible,

\footnotetext{
${ }^{4}$ For those studies that used the CBU volunteer panel, we took care that no participant was involved in more than one study.
} 
without touching or crossing lines, entering parts of the maze blocked at one end, tracing over the maze, or lifting the pen off the paper, until the maze had been completed. Examples of each of these violations were provided by the experimenter on a separate maze. A qualitative (Q) score was calculated in line with Porteus's (1965) procedure. The Q score reflected the number of violations made by each participant, with higher $\mathrm{Q}$ scores equating to more violations.

Beck Depression Inventory (BDI; Beck, Ward, Mendelson, Mock, \& Erbaugh, 1961). The BDI is a 21-item inventory presented in multiplechoice format that measures presence and degree of depression. Each item corresponds to a specific category of depressive symptom and/or attitude and consists of a graded series of four self-evaluative statements that are rank ordered and weighted to reflect the range of severity of the symptom from neutral to maximum severity $(0-3)$. The psychometric properties of the BDI are good (Beck, Steer, \& Garbin, 1988). The BDI was used for all subsequent studies (3-8) as the measure of depressed mood.

\section{Procedure}

Because of the number of tests administered, counterbalancing was not feasible. The AMT was therefore administered first to avoid the possibility of the other tests influencing the memories recalled. All participants completed the tests in the following order: AMT, verbal fluency, Block Design, design fluency, Porteus Maze, Alternate Uses Test, and BDI.

\section{Results and Discussion}

The mean scores for the 24 participants on the different measures are reported in Table 2. To examine the hypothesis that AMS would be associated with scores on the tests loading on executive control, we performed a series of partial correlational analyses with self-reported depression (on the BDI), which correlated significantly with AMS, $r(22)=-.69, p<.001$, partialed out.

AMS did not partially correlate significantly with any of the measures of executive control that were corrected for errors (verbal fluency, Alternate Uses Test, design fluency), $\operatorname{prs}(21)<|.26|$, $p s>.22$. The only significant correlations were with the Porteus Maze Q score (a measure of errors), $\operatorname{pr}(21)=-.50, p<.05$, and the WAIS-III Block Design score, which incorporates a measure of errors, $\operatorname{pr}(21)=.42, p<.05$.

Table 2

Demographic, Self-Reported Depression, Memory, and Executive Control Task Data in Study 2

\begin{tabular}{lrc}
\hline \multicolumn{1}{c}{ Variable } & $M$ & $S D$ \\
\hline Age (years) & 40.83 & 9.18 \\
Verbal fluency_correct & 13.13 & 3.62 \\
Verbal fluency_error & 0.58 & 1.56 \\
Block Design (scaled score) & 12.17 & 3.91 \\
Design fluency—correct & 8.33 & 3.23 \\
Design fluency_error & 0.29 & 0.62 \\
Alternate Uses Test—correct & 4.92 & 1.59 \\
Alternate Uses Test-error & 0.29 & 0.69 \\
Porteus Maze Test Q score & 5.29 & 6.74 \\
Beck Depression Inventory & 13.83 & 9.89 \\
No. specific memories on the AMT & 16.79 & 5.32 \\
Total errors & 1.17 & 1.97 \\
\hline
\end{tabular}

Note. $n=24$ (5 men, 19 women). AMT = Autobiographical Memory Test; Total errors = sum of errors in design fluency, verbal fluency, and on the Alternate Uses Test.
On this basis, we decided a posteriori to examine the relationship between AMS and the sum of the error scores across the measures that did not already index errors (i.e., sum of errors on the Alternate Uses Test, design fluency, and verbal fluency; see Table 2). AMS was also significantly correlated with this combined error score, with BDI scores partialed out, $\operatorname{pr}(21)=-.51$, $p<.02$, indicating that having a greater number of errors across the range of executive control tasks was associated with reduced AMS.

To examine this relationship with errors further, we conducted a multiple linear regression with AMS as the dependent variable. BDI scores were entered on Step 1 and accounted for approximately $47 \%$ of the variance in AMS, $F(1,22)=19.80, p<.001$. Next, the number-correct scores on the different tasks (verbal fluency, design fluency, and Alternate Uses Test) were entered on Step 2 and uniquely, but nonsignificantly, accounted for $7 \%$ of the AMS variance $(F<1)$. Finally, the summed error score for these measures along with the Porteus Maze Q score (a measure of errors) were entered on Step 3 and uniquely and significantly accounted for $19 \%$ of the AMS variance, $F(2,17)=5.95, p<.05$.

Overall, this pattern of data suggests that those processes that drive AMS performance are to some extent related to the processes that allow errors on a variety of measures of executive control. On this point, it is important to acknowledge that the number of specific memories retrieved on the AMT (i.e., level of AMS) is a direct reflection of the number of "errors" on the AMT (as the sum of number-correct scores and errors is a constant across participants). For instance, producing 17 specific memories to 24 word cues on the AMT reflects the fact that to 7 of the cue words the participant failed to generate a specific memory within the time limit. However, the same relationship does not hold for several of the executive control tasks in the present study (Alternate Uses Test, Porteus Maze, verbal fluency, design fluency), in which the numbers of correct responses and errors are not arithmetically related in any straightforward way. In contrast, the score for WAIS-III Block Design does reflect the number of errors as it represents the number of designs (out of a fixed total) that the participant was able to reproduce within certain constraints.

As AMS scores directly reflect the number of errors on the AMT and correlate significantly only with those aspects of executive control tests that also directly reflect numbers of errors, one way to conceptualize the present pattern of data is that AMS scores, at least in part, are a proxy measure of some form of tendency to generate errors, and/or a failure to prevent errors, on executive-control-demanding tasks. This account may partly explain the current lack of clear findings in the AMT literature concerning the relationship between AMS and executive functioning (see the introduction) in that it may be that many of the relevant studies have ignored error scores. This focus would lead to variability in the pattern of data as a function of the extent to which the number-correct scores directly reflected the numbers of errors for given participant groups and/or executive control tasks. For instance, differences in the relationship between numbercorrect scores and error scores across the samples could explain why we found a clear relationship between verbal fluency numbercorrect scores and AMS in Study 1 yet a much weaker relationship in Study 2.

However, it is important to note that the present analyses regarding errors were post hoc and are therefore in need of replica- 
tion. In addition, the numbers of errors on the chosen tasks in the present study were small, leading in most cases to problems with using parametric analyses for the tasks individually (hence the use of the combined error score). We therefore sought a conceptual replication of these findings in Study 3, this time with the a priori hypothesis that, independent of depression, reduced AMS would be associated with errors on another cognitive task requiring executive control, which nevertheless again had little to do with autobiography and emotion.

\section{Study 3: Performance on the AMT and a Number- Generation Task}

In Study 3 we sought to examine the relationship between AMS and error performance on an executive control task which, as with some of the earlier executive control tasks, made no explicit demands on autobiographical memory. For this study we wanted versions of the AMT and of another task that would both generate significant proportions of errors in unselected participants to facilitate correlational analyses. We therefore devised an "extended time" version of the AMT in which all of the cue words referred to periods of time longer than 1 day (e.g., bereavement, holiday). We reasoned that it would be more difficult to generate specific memories of events lasting less than a day to such cues than on the standard version of the AMT and therefore that the numbers of nonspecific memories (i.e., errors) would be greater, leading to lower AMS scores. For the comparison executive control task we selected a number-generation paradigm that had been devised previously (Scott, Barnard, \& May, 2001a, 2001b), in which participants generate numbers that satisfy a variety of different task constraints (e.g., " a sequence of six numbers within the range two hundred and two up to five hundred and ninety-eight"). Existing research on this number-generation task indicated that participants often make mistakes that reflect a failure to satisfy one of the task constraints while meeting the other constraints successfully (e.g., coming up with a sequence of six numbers that nevertheless falls outside the specified range; Scott et al., 2001a, 2001b). This state of affairs seems analogous to the task parameters of the AMT, in which correct responses (specific memories) must satisfy a number of constraints (i.e., they must be autobiographical, a memory, and of an event lasting less than 1 day). Our hypothesis for Study 3 was therefore that AMS would be significantly negatively correlated with numbers of errors on this number-generation task, independent of self-reported depression.

\section{Method}

\section{Participants}

Twenty-four participants (7 men, 17 women) were recruited from the $\mathrm{CBU}$ volunteer panel. The mean age was 35.26 years $(S D=11.12$ years, range $=19-51$ years $)$. Mean BDI score was $7.78(S D=6.16)$.

\section{Measures and Procedure}

The AMT. The AMT was as for Studies 1 and 2. On this occasion 15 words were used ( 5 negative, 5 positive, and 5 neutral) with three practice words (gigantic, grass, and absence). As noted above, we selected cue words that referred to periods of time lasting longer than 1 day on the assumption that it might be more difficult to retrieve specific memories lasting less than 1 day to such cues (e.g., holiday, bereavement, adolescence). The process of selecting these words is described in detail in the Method section of Study 6.

Number-generation task. Fifteen questions were chosen from Scott et al.'s (2001a, 2001b) studies. All of the questions required participants to generate a number that had to satisfy two or more constraints (e.g., "Could you please give me a seven-figure number between two million and ten million?" "Could you please give me a sequence of any three numbers within the range one hundred and one up to four hundred and ninetynine?"). The fifteen items with the highest mean error rates according to Scott et al. (2001a, 2001b) were selected in order to preclude floor effects on the task. The questions were presented verbally as per Scott et al.'s (2001a, 2001b) study. Participants were given three simple numbergeneration questions as practice. They were also told that the experimenter would present each question once and would not repeat it. The errors on the task were of three types: numbers not in sequence, an incorrect number of digits, and numbers that fell outside the upper or lower bounds. These three types of error were summed to provide an overall error score. The AMT and the number-generation task (in counterbalanced order) were carried out first, followed by the BDI.

\section{Results and Discussion}

One participant's data had to be set aside because of problems with the tape recorder during the AMT task. AMS $(M=9.57$, $S D=3.69$ ) was significantly negatively correlated with number task error scores $(M=4.00, S D=3.68), \operatorname{pr}(19)=-.39, p<.05$, with both self-reported depression on the BDI and age partialed out (both self-reported depression on the BDI and age showed a significant correlation with AMS: BDI, $r(21)=-.38, p<.05$; age, $r(21)=-.40, p<.05)$. These results supported our hypothesis that reduced AMS would be associated with more errors on the number task.

Together with the data from Studies 1 and 2, the present results provide support for the view that AMS scores reflect a tendency to generate errors across a range of different executive control tasks. What might be the candidate explanations for how this view of AMS relates to the notion of executive control?

At least two possibilities merit serious consideration. The first is that successful performance of the different executive control tasks that have been examined requires effective inhibition of inappropriate candidate task responses and/or behavioral impulses. So, for instance, on the number-generation task the process of searching for numbers meeting all of the task requirements is likely to bring to mind candidate responses that fall foul of one or more of those requirements (e.g., a number that has the correct number of digits but falls outside of the specified range). To carry out the task correctly, the participant needs to inhibit these candidate responses and continue searching for a number that meets all of the task parameters. Errors on the task occur when such inhibition is unsuccessful and the candidate responses consequently become actual responses on the task. In line with this type of inhibition account, it is notable that the types of errors that participants have made on the tasks studied so far have almost always been task related in the way that one would predict. So, for instance, errors on the number-generation task did indeed consist of numbers that met one or more (but not all) of the task parameters. Likewise, errors in verbal fluency were usually not words beginning with the wrong letter but rather repetitions of previously generated words, proper nouns beginning with the correct letter, or words that share 
the same stem as previous responses (e.g., swim, swimming, swim$m e r$, all of which violate the specific task instructions.

A similar explanation can be proposed for the AMT, in which, we suggest, the memory search process is likely to generate candidate responses in the form of overgeneral memories or semantic associates that need to be inhibited in the search for an appropriately specific response. A breakdown in such inhibition would result in these candidates being proffered as actual responses, thus leading to reduced AMS on the task.

An alternative possibility is that errors on the various executive control tasks under consideration reflect an inability to hold all of the task parameters in mind for the duration of the task. Consequently, inappropriate candidate task responses are filtered through an incomplete representation of the task parameters and hence form the basis of overt error responses.

Both of these possibilities are commensurate with the sort of executive control account of cognitive processing in depression that was outlined in the introduction (e.g., Hertel, 2000). In other words, executive control deficits lead to either poor inhibition of inappropriate target responses and/or poor "online" application of the complete set of task instructions. Furthermore, this type of account is not dissimilar to models of executive control in the mainstream cognitive psychology literature: for example, the controlled attention model of Engle and colleagues (e.g., Engle, 2002; Engle \& Kane, 2004; for a review, see Barrett et al., 2004). The core feature of this particular approach is that individual differences in executive control, labeled in this framework working memory capacity (WMC), reflect the availability of a domain-free controlled attentional resource that is necessary to maintain temporary goals (e.g., those pertaining to executive task performance) in the face of distraction or interference (e.g., from inappropriate candidate task responses). WMC is only one of several related such notions in the cognitive psychology literature and maps onto what Baddeley called the central executive (Baddeley, 1983, 1986), to what Norman and Shallice (1986) called the Supervisory Attentional System, and to what Posner and Snyder (1975) and Schneider and Shiffrin (1977) called controlled attention (see Miyake \& Shah, 1999). Similarly, the argument that task errors in which one or more, but critically not all, task demands are met, bears similarity to notions such as goal neglect in the work of Duncan and colleagues (Duncan, Emslie, \& Williams, 1996; Kane \& Engle, 2003).

Fluid intelligence, or the ability to solve novel problems, has been shown to be strongly correlated with measures of constructs such as WMC and degree of goal neglect in studies in the mainstream cognitive psychology literature (A. R. A. Conway, Kane, \& Engle, 2003; Duncan et al., 1996; Engle, Tuholski, Laughlin, \& Conway, 1999; Unsworth \& Engle, 2005). Fluid intelligence is nonverbal and to a large extent culture free (Horn \& Cattell, 1967). If AMT performance is dependent on problems with executive control as outlined above and as reflected in notions such as WMC and goal neglect, then AMS scores should correlate with measures of fluid intelligence, independent of depression. Given the equivocal nature of the existing research on AMS and measures of nonfluid intelligence (e.g., Park et al., 2002) this seems an important test of an executive control view of reduced AMS.

In Study 4 we therefore sought to investigate this predicted relationship using Cattell's Culture Fair Test of "g" (Cattell \& Cattell, 1960)—a standard measure of fluid intelligence. Our hy- pothesis was that there would be a significant correlation between reduced AMS and lower levels of $\mathrm{g}$, independent of depression.

\section{Study 4: Performance on the AMT and Cattell's Culture} Fair Test of Fluid Intelligence (Spearman's ' $g$ ”)

\section{Method}

\section{Participants}

We carried out a small pilot study in which we obtained Cattell "g" scores from the CBU volunteer panel records (on the basis of previous completion of the test) for 10 panel participants who had completed the AMT in an unrelated study. For this pilot sample, the correlation between AMS and $\mathrm{g}$ scores was very high, $r(8)=.72, p<.01$. On this basis we performed a power analysis to provide $80 \%$ power that a correlation of $r=$ .60 would be greater than zero, with alpha set at .05 . This indicated a sample size of 16 or greater (Cohen, 1988). Consequently, 20 unselected participants (13 women, 7 men) were recruited from the CBU volunteer panel. The mean age of the participants was 40.65 years $(S D=17.23$ years, range $=17-60$ years $)$. The mean BDI score was $5.45(S D=4.85)$.

\section{Measures and Procedure}

The AMT. The AMT for this study was as for Studies 1-3. It consisted this time of the 18 low-imageability cue words used by Williams, Healy, and Ellis (1999). This is because low-imageability cues have been found to lead to relatively fewer specific memories. Using those cues was therefore likely to lead to a greater range of AMS scores, which would facilitate correlational analyses.

Cattell's Culture Fair Test of " $g$ ”-Scale 2 Form A (Cattell \& Cattell, 1960). The Culture Fair Test of "g," or fluid intelligence, contains 50 items, organized into four nonverbal tasks. It is designed to minimize the influence of verbal comprehension, education level, and culture on various aspects of the evaluation procedure, such as the administration instructions and the item content. In the first subtest (series), individuals are presented with an incomplete, progressive series of figures. The participants' task is to select, from among the choices provided, the answer that best continues the series. In the second subtest (classification), participants are presented with five figures and must select the one that is different from the other four. In the third subtest (matrices), the task is to correctly complete the matrix presented at the left of each row. Finally, in the fourth subtest (conditions), participants select a figure that duplicates the topological relationships of a target design. Participants completed the AMT first, followed by Cattell's Culture Fair Test of "g" and the BDI.

\section{Results and Discussion}

The hypothesis that AMS scores $(M=11.75, S D=5.46)$ would be significantly positively correlated with Cattell "g" scores $(M=$ 34.60, $S D=5.62)$, even after partialing out self-reported depression on the BDI-which this time did not correlate significantly with AMS, $r(18)=-.14, n s$-was supported, $\operatorname{pr}(17)=.60, p<$ .01. This finding provides further support for the view that reduced AMS indexes some form of executive control deficit, as such deficits are closely indexed by fluid intelligence (Engle et al., 1999; Kane \& Engle, 2002).

\section{Interim Summary of the First Four Studies}

Our overarching aim behind the present series of studies was to investigate the extent to which performance on the AMT, particularly in depressed individuals, is a function of reduced executive 
control associated with depressed mood (Ellis \& Ashbrook, 1988; Hartlage et al., 1993; Hasher \& Zacks, 1979; Hertel \& Hardin, 1990). The first stage of this examination involved demonstrating that the AMT is indeed an executive-control-demanding task, independent of any relationship with depressed mood. Thus, in the four studies up to this point we have shown a clear relationship between performance on the AMT and on other nonautobiographical and generally emotion-unrelated tasks that depend on executive control. In doing so we have exploited naturally occurring individual differences in executive control to establish the existence of such relationships independent of levels of depressed mood. Furthermore, we have indicated that the clearest relationship across tasks involved error scores, and we have speculated in the Results and Discussion section of Study 3 about how deficits in executive control may have led to such correlated patterns of errors across tasks.

Finally, we have shown in Study 1 that a measure of executive control (verbal fluency) significantly mediates the relationships between both depressed mood and an eating disorder diagnosis (as predictors) and AMS as the criterion, thus providing evidence that variations in executive control can significantly account for the relationship between mood or psychopathology and AMS. We do not report mediational analyses from Studies 2-4, as these studies were on unselected participants and so it is inappropriate to analyze the data in terms of hypotheses based on the effects of significant depressed mood or other forms of psychopathology.

Having established that the AMT is a task susceptible to variations in executive control independent of depression and that executive control mediates the relationships between depression or psychopathology and AMS (Studies 1-4), the next challenge was to gather experimental evidence that the diminished executive control associated with depressed mood is an important factor in determining the patterns of AMT performance in depression. To this end, we sought to manipulate the task parameters of the AMT in ways that would interact with individual differences in executive control in order to show that these manipulations can interact in the same way with individual differences in levels of depressed mood. In Studies 6-8 we examined three such manipulations using stratified samples of participants selected so as to have a broad range of self-reported depression severity. However, first we wanted to demonstrate (in Study 5) that AMS on the standard version of the AMT does indeed correlate with levels of depressed mood in such a stratified sample, as this is obviously a prerequisite of any studies that seek to manipulate the parameters of the standard AMT to influence a putative relationship between AMS and depressed mood within such samples.

\section{Study 5: The Standard Version of the AMT and Its Relationship to Depressed Mood}

Although meta-analysis has indicated a large correlation (Cohen, 1988) between reduced AMS and elevated self-reported depression scores in the existing literature, as outlined in the introduction to this article (van Vreeswijk \& de Wilde, 2004), many of the relevant studies have involved clinical groups. Fewer studies have examined this link outside of the clinical literature (e.g., Ramponi et al., 2004). As Studies 6-8 examined self-reported depressed mood and AMS performance in nonclinical samples, it seemed useful and important to first verify the existence of a relationship between depressed mood and AMS in such nonclinical groups. To this end, Study 5 simply involved administering the AMT with a standard word set to a large nonclinical sample stratified so as to include a wide range of self-reported depression scores (on the BDI). Of course, the data from Studies 1-4 in the present series are able to speak to this issue, as each involved a measure of depressed mood and a version of the AMT. The average correlation (using a $z$-transformation method) between AMS and depressed mood from this initial tranche of studies was $r=-.43$ (Corey, Dunlap, \& Burke, 1998). However, although this is encouraging, in these first four studies the samples were not selected on the basis of depression scores, thus it was important to benchmark the same effect in a sample stratified on depression before examining AMT task manipulations in similarly stratified samples (Studies 6-8).

\section{Method}

For the present study (and for Studies 6-8) we used a stratified approach to recruit participants with a wide range of scores on the BDI to facilitate correlational analyses involving depressed mood. To obtain this range of depression scores we selected participants on the basis of their BDI scores from previous studies in the CBU. We sourced representative numbers of participants classified as "nondepressed" (BDI scores of 0-9), "mildly to moderately depressed" (10-23), and "severely depressed" (24-63) on the basis of these previous BDI scores (Shaw, Vallis, \& McCabe, 1985). On this basis, 60 participants (44 women, 16 men) were selected for the present study, with a close to maximal range of BDI scores $(0-58$; the maximum range is in fact $0-63$ ), with $n=25$ in the nondepressed range (mean age $=39.92$ years, $S D=10.19$ years; 19 women, 6 men), $n=10$ in the mild-to-moderate range (mean age $=35.50$ years, $S D=12.29$ years; 5 women, 5 men), and $n=25$ in the severe range (mean age $=38.84$ years, $S D=10.47$ years; 20 women, 5 men). Mean overall age was 38.73 years $(S D=10.60$ years, range $=20-58$ years $)$.

The AMT methodology was as for Studies 1-4. This time 14 cue words (7 positive and 7 negative) were selected from Brittlebank et al.'s (1993) study. The positive and negative sets were balanced for emotionality and frequency using the Kučera and Francis (1967) norms. Two practice words were used, exhausted and interested. The AMT was carried out first, followed by the BDI.

\section{Results and Discussion}

In line with our hypothesis, self-reported depression on the BDI $(M=19.85, S D=17.33)$ showed a moderate negative correlation with levels of AMS $(M=6.15, S D=4.07), r(58)=-.41, p<$ .01. These data in a stratified sample, along with those from published studies (e.g., Ramponi et al., 2004; van Vreeswijk \& de Wilde, 2004) and the data on unselected participants from Studies $2-4$, suggest that elevated levels of depressed mood on the BDI in analogue and subclinical samples are moderately negatively correlated (Cohen, 1988) with reduced AMS on the standard AMT. This replicated AMS-depression effect thus provides a platform from which we can manipulate the parameters of the standard AMT (Studies 6-8) to examine putative effects on the AMSdepression relationship.

\section{Study 6: Short-Duration Versus Long-Duration Words on the AMT}

A clear prediction from the arguments outlined so far is that if depression is associated with impoverished executive control (see 
Hertel, 2000), those participants with higher levels of depression should find it harder to perform the AMT when the task parameters are manipulated such that there is a greater requirement to offset or inhibit the influence of distracting information (Barrett et al., 2004). To examine this issue we therefore devised a version of the AMT with two types of cue words. Half of the cue words were chosen with the intention of priming distracting information in the form of candidate AMT responses that do not fit the task demands. The remaining half of the cue words were chosen with the intention of minimizing the priming of such distracting information. To this end, the former half of the cue words related to periods of time that were longer than 1 day (e.g., summer, cancer, adolescence; see Study 3). The rationale was that such cue words would, in a significant proportion of cases, automatically prime candidate autobiographical memories that related to periods of time longer than 1 day. Therefore, to perform the AMT successfully (i.e., to generate memories that refer to events lasting less than 1 day), the tendency to produce these candidate memories as task responses would need to be inhibited and the retrieval search process continued. Our prediction was that for these long-duration words higher levels of depressed mood should be associated with more frequent failures to inhibit such response tendencies successfully, thus leading to reduced AMS.

In contrast, the second set of cue words all referred to events that routinely last less than 1 day (e.g., kiss, accident, evening). Our rationale was that such cue words would automatically prime far fewer inappropriate candidate memories (e.g., overgeneral memories). Instead, they should prime candidates that would be far more likely already to meet the task demands of the AMT (i.e., specific memories). Our prediction here was that there would be little or no correlation between AMS on this short-duration word set and levels of depressed mood, as there should be a minimal requirement to inhibit inappropriate task-generated response tendencies. Critically, we hypothesized that an index of the difference in AMS scores between the long-duration words and the shortduration words would correlate with levels of depressed mood, indicating that as mood increased this putative manipulation of levels of to-be-inhibited response tendencies would have a greater impact on AMS.

Our three hypotheses were therefore as follows:

Hypothesis 1: Levels of AMS will be higher to short-duration words than to long-duration words.

Hypothesis 2: An index of the difference in AMS scores for long- versus short-duration words will correlate with BDI scores, with increasing BDI scores relating to a relative reduction in AMS to long-duration words compared with shortduration words.

Hypothesis 3: BDI scores will show a negative correlation with AMS for the long-duration words, but there will be little or no correlation between AMS and BDI scores for the short-duration words.

\section{Method}

\section{Participants}

Eighteen participants (10 women, 8 men) were recruited from the CBU volunteer panel with a range of depression scores $(0-28 ; n=11$ in the mild range, mean age $=34.45, S D=10.61,8$ women, 3 men; $n=5$ in the mild-to-moderate range, mean age $=29.50, S D=10.88,2$ women, 3 men; $n=2$ in the severe range, mean age $=39.00, S D=9.90,0$ women, 2 men; Shaw et al., 1985; see Study 5). ${ }^{5}$ The mean age of the participants was 33.82 years $(S D=10.34$ years, range $=21-49$ years $)$.

\section{Materials and Procedure}

The AMT. The AMT was as for Studies 1-5 except that this time it consisted of 30 words (5 positive, long duration, holiday, love, prosperity, romance, summer; 5 negative, long duration, bereavement, cancer, depression, disease, slavery; 5 neutral, long duration, adolescence, century, eternity, past, permanent; 5 positive, short duration, dance, kiss, laugh, party wedding; 5 negative, short duration, accident, attack, cry, quarrel, sneer; and 5 neutral, short duration, evening, moment, movie, occasion, utterance). All words were selected from the Medical Research Council Psycholinguistic Database-Version 2.00 (Wilson, 1987). Initially a list was generated of those 4,852 words that had full data regarding concreteness, part of speech, familiarity, imageability, Kučera-Francis written frequency (Kučera \& Francis, 1967), and number of letters. Tim Dalgleish and Cecilia $\mathrm{Au}$ Yeung independently selected long-duration and shortduration words from the list that represented, respectively, short (1 day or less) versus long (more than 1 day) periods of time. After consultation, this resulted in unanimous agreement on a list of 187 words.

This reduced list of 187 words was then given to four people to be rated independently in terms of the valence (positive, negative, or neutral), time duration (short, long, or not sure), and emotionality (a 7-point scale ranging from 1 [no emotional association] to 7 [strong emotional associations]) of each word. Examples and explanations of each rating were given. The final sets of 15 long-duration and 15 short-duration words were selected such that they were comparable on emotionality, frequency, imageability, and number of letters (words were also matched on these dimensions across valence categories). For the purpose of the present analyses we computed an index of the difference between AMS performance on short-duration versus long-duration words (AMS to short-duration words minus AMS to long-duration words). The AMT was carried out first, followed by the BDI

\section{Results and Discussion}

Participant characteristics and AMS data for the short-duration and long-duration words as well as the index of the difference between short-duration and long-duration word performance are presented in Table 3. In line with the first hypothesis, participants showed reduced AMS to long-duration words compared with short-duration words, $t(17)=8.17, p<.001, d=2.04$. In line with the second hypothesis, there was a significant correlation between BDI scores and the index of the difference in AMS for short-duration and long-duration words, $r(16)=.55, p<.02$, with greater depression being associated with a greater tendency for long-duration words, relative to short-duration words, to reduce AMS. Finally, in line with the third hypothesis, BDI scores showed a negative correlation with AMS to long-duration words, $r(16)=$ $-.50, p<.05$ (thus further replicating the relationship between depressed mood and reduced AMS) but almost no correlation with AMS to short-duration words, $r(16)=-.06, p>.80$.

These results indicate that manipulating the nature of the cue words on the AMT with the aim of introducing putative variations

\footnotetext{
5 A power analysis based on the anticipated large correlations (Cohen, 1998) indicated a sample size of 20 or more, so the present study was slightly underpowered. This was due to recruitment difficulties at the time of the research.
} 
Table 3

Demographic, Self-Reported Depression, and Memory Data in Study 6

\begin{tabular}{lrc}
\hline \multicolumn{1}{c}{ Variable } & $M$ & $S D$ \\
\hline Age (years) & 33.82 & $10.34^{\mathrm{a}}$ \\
Beck Depression Inventory & 8.78 & 7.46 \\
No. specific memories on the AMT: short-duration words & 12.56 & 2.33 \\
No. specific memories on the AMT: long-duration words & 7.44 & 2.68 \\
No. specific memories on the AMT: short- minus long-duration words & 5.11 & 2.65 \\
\hline
\end{tabular}

Note. $n=18$ ( 8 men, 10 women).

${ }^{\text {a }}$ Data from 1 participant were not available.

in levels of primed task-inappropriate candidate responses can bring about variations in the strength of the relationship between depressed mood and AMS. This suggests that reduced executive control associated with depressed mood is important in driving the relationship between depression and AMS. Study 7 involved a different form of AMT task manipulation designed to influence the relationship between depressed mood and AMS - the addition of mental load.

\section{Study 7: The Interaction of Depressed Mood and Mental Load on the AMT}

In many cases, individuals with low levels of executive control are arguably more susceptible to manipulations of mental load in dual-task experiments because they have poorer control capabilities to begin with (Barrett et al., 2004). On the basis of the view that depressed mood levels are to some extent associated with variations in executive control, one would predict that higher levels of depressed mood would be associated with greater susceptibility to such load manipulations. On this basis we asked participants with varying levels of depressed mood to carry out two versions of the AMT in a within-subjects design, with and without the mental load of holding in memory a list of digits for later recall. Our hypothesis was that an index of the difference in AMS scores (cf. Study 6) between the load and no-load conditions of the AMT would correlate with depressed mood on the BDI, with higher BDI scores being associated with lower AMS under load, relative to no load.

\section{Method}

\section{Participants}

Power analysis based on the correlation between depressed mood and the index of AMS performance across long-duration and short-duration words from Study 6 indicated a sample size of 20 or more to give $80 \%$ power that a given correlation would differ from zero in a particular direction with alpha set at .05 . Twenty-three participants (14 women, 9 men) were therefore recruited from the $\mathrm{CBU}$ volunteer panel, again with a range of scores on the BDI $(0-31 ; n=11$ in the mild range, mean age $=$ 34.64, $S D=15.19,8$ women, 3 men; $n=9$ in the mild-to-moderate range, mean age $=33.56, S D=13.78,4$ women, 5 men; $n=3$ in the severe range, mean age $=33.00, S D=12.29,2$ women, 1 man; Shaw et al., 1985; see Study 5$)$. The mean overall age was 34.00 years ( $S D=13.72$ years).

\section{Materials and Measures}

The AMT. The AMT was broadly the same as in the previous studies. In this version, two matched sets of 16 words were chosen. To maximize the range of AMS scores for the purposes of correlational analyses, we drew these words from the list of long-duration words described in Study 6 and from the list of low-imageability words used in Williams, Healy, and Ellis's (1999) study, which indicated that reduced AMS was associated with low imageability of the cue words in healthy volunteers. There were 8 positive and 8 negative words in each set. The two sets were comparable on frequency, imageability, and emotionality. Word List A comprised the following: bereavement, cancer, century, eternity, explanation, knowledge, law, obedience, past, permission, romance, situation, slavery, soul, upkeep, and winter. Word List B comprised the following: adolescence, boredom, depression, disease, duty, effort, hearing, holiday, interest, legislation, love, mood, opportunity, permanent, prosperity, and summer.

WAIS-III Digit Span. Each participant's forward digit span was measured using the Digit Span subtest of the WAIS-III. This involves presenting participants with a series of number sequences of increasing length. Participants' digit span was indicated by the highest sequence repeated correctly.

\section{Procedure}

Each participant performed the AMT twice, once under a condition of mental load and once with no load. In the load condition participants were presented with a sequence of digits prior to each cue word on the AMT. The length of the string of digits was 2 less than the participants' digit span. Participants were instructed to remember the digits for recall after retrieval of a memory to the subsequent AMT cue word. The digits were read aloud to the participant before the cue word was presented and were recalled after the participant had given a memory or after $2 \mathrm{~min}$ had passed, in the event that no memory was generated. Approximately half of the participants $(n=$ 12) carried out the AMT under mental load with Word List A, and the rest $(n=11)$ carried out the AMT with Word List B. Order of presentation of the two AMT conditions (load, no load) was also approximately counterbalanced across participants (load then no load, $n=11$; no load then load, $n=12$ ).

The WAIS-III Digit Span subtest was administered first, followed by the two versions of the AMT. Participants then completed the BDI.

\section{Results and Discussion}

Mean raw digit span across participants was $7.52(S D=1.04)$. To examine our hypothesis that depressed mood (on the BDI) would be associated with an increased tendency toward reduced AMS under mental load (versus no load), we computed an AMS index by subtracting AMS scores in the load condition $(M=$ $13.35, S D=2.48)$ from those in the no-load condition $(M=13.70$, $S D=2.64)$. There was a significant correlation between BDI scores $(M=10.04, S D=8.14)$ and this AMS index $(M=2.00$, $S D=1.00), r(21)=.42, p<.05$, in support of the hypothesis.

This provides further support (along with Study 6) that manipulating the AMT parameters in ways that we know would interact 
with individual differences in executive control can influence the relationship between depressed mood and AMS. This again suggests that diminished executive control associated with depressed mood can to a significant extent drive the relationship between depression and AMS.

We were concerned that, although the correlational aspects of the data were as we predicted, examination of the scatter plot of the AMS index and BDI scores revealed that a number of participants with low scores on the BDI had a negative AMS index. This indicates that AMS scores were higher under conditions of load, compared with no load. This would account for why the mean AMS scores for the load and no-load conditions were relatively similar.

This finding suggests that the load applied by remembering numbers may not have compromised executive control sufficiently for those with greater executive control capabilities (i.e., those lower in depressed mood). It is possible that under conditions of mental load, these participants were sufficiently able to increase their level of controlled attention (Barrett et al., 2004) during the AMT to compensate (or even overcompensate) for the effects of the load. To investigate this hypothesis more systematically, we performed a second mental load study using the same methodology as in Study 7, this time exclusively with participants with low BDI scores $(N=16$; BDI $<10)$, who we expected to have relatively unimpaired executive control. Replicating the results for low-BDI participants in Study 7, these new low-BDI participants were significantly less specific under conditions of no load $(M=11.50, S D=3.20)$ as compared with conditions of load $(M=12.81, S D=1.97), t(15)=2.31, p<$ .05 , one-tailed.

However, a further concern was that mental load might be associated with relatively increased AMS in low-depression participants, not because of the load per se, but because in the load condition the AMT task was effectively interrupted by presentation of the to-be-recalled digits and by their later recall. In contrast, in the no-load condition there was no such interruption of the AMT. It may therefore have been the case that after each interruption in the load condition the low-BDI participants with their relatively unimpaired executive control were able to refocus on their mental representation of the AMT task instructions to remind themselves of what they had to do-in particular, the need to keep their responses specific. For these participants, this may then have served to make responses on the load version of the AMT more specific (higher AMS) than on the no-load version as a function of this continued "revision" of the task instructions.

To examine this possibility we carried out a second supplementary mental "load" study in which exclusively low-BDI participants $(N=20$; BDIs $<10)$, in a within-subjects design, were either interrupted during the AMT and given a digit span for immediate recall (i.e., there was no load) or not interrupted and completed the AMT straight through. We used the same AMT words as in the previous supplementary study. The data provided no support for the view that interruption per se was responsible for the load-no load differences in low-BDI participants reported above, as there was no significant difference between the interruption and no-interruption conditions, $t(19)=0.78, p=.45$.
Study 8: The AMT With Reversed Instructions (AMT-R) and Its Relationship to Depressed Mood

A key aspect of the argument developed throughout this article-that levels of AMS on the AMT are to a significant extent a function of executive control capabilities-is that AMT performance requires sustained use of controlled attention to retrieve specific memories, maintain task instructions, and deal with the influence of automatically generated candidate memory response tendencies that do not meet the AMT task requirements (i.e., they are not specific). If such executive control is indeed a critical explanatory variable with respect to AMT performance, then there should be nothing particularly special about specific memories as far as the executive control contribution to AMS is concerned. Within an executive control analysis, specific memories simply represent correct performance on the AMT as it is normally constrained. Consequently, it should be possible to produce a mirror image of the standard reduced AMS effect in depression by asking participants to generate nonspecific (i.e., general) autobiographical memories in a "reversed" version of the AMT (which we shall call the AMT-R). In the AMT-R, the cue words should automatically prime a proportion of specific candidate memory response tendencies, which need to be inhibited so that the participant can continue to search the autobiographical database for suitably general candidates. An executive control analysis of the AMT-R would therefore predict that depressed mood would be correlated with greater AMS, as AMS would be an index of AMT errors. It is important to note here that the prediction is not that participants with higher levels of depressed mood will be more specific on the AMT-R than they routinely are on the standard AMT. Rather, the prediction is that on the AMT-R, those with higher levels of depressed mood would be more specific than those with lower levels of depressed mood.

One would perhaps not expect the absolute magnitude of this relationship between AMS and depressed mood on the AMT-R to be as strong as on the standard version of the task. The reason for this is that autobiographical memory search is generally regarded as hierarchical, with generic autobiographical descriptions such as "lifetime periods" at the top of the hierarchy and specific memories at the bottom (e.g., M. A. Conway \& Pleydell-Pearce, 2000). In the AMT-R, to-be-inhibited candidate memories are likely to include only those specific memories activated by some process of direct retrieval that bypasses hierarchical search (M. A. Conway \& Pleydell-Pearce, 2000). In contrast, in the standard version of the AMT the pool of inappropriate candidate memories includes all those activated as the retrieval process moves down the hierarchy. This difference means that the standard version of the AMT is likely to put greater demands on executive control processes (as more response tendencies will require inhibition) than is the AMT-R. Consequently, one would predict that the absolute values of any correlations between AMS and depressed mood on the AMT-R would be lower than those we found in the previous studies using the standard AMT (with the benchmark being the moderate correlation in Study 5).

As we have noted earlier, this analysis of the AMT in terms of the influence of executive control is somewhat different from the extant theoretical proposals in the literature on AMS. Here, specific memories often have something of a privileged status as they carry detailed information about the emotional past that may be 
potentially distressing. As discussed in the introduction to this article, the affect regulation hypothesis (Williams, 1996) therefore proposes that reduced AMS on the standard AMT is not wholly a function of the task parameters but represents a memory retrieval style that has developed to avoid retrieving this detailed distressing information. The proposal is that this avoidant style is associated with greater levels of depressed mood and psychopathology, in particular clinical depression and a history of trauma (e.g., Hermans et al., 2004). To the extent that reduced AMS is performing an affect regulation function, one would predict that reversing the AMT instructions would not reverse the direction of the relationship between depressed mood and AMS as, regardless of the instructions of the task, higher levels of depression should still be associated with a greater need for affect regulation and thus with lower levels of memory specificity.

We examined these issues in Study 8. For the AMT-R, participants were asked to generate categorical memories to cue words, that is, memories that conflated across more than one specific occasion (so, to the cue word party, a categorical response might be "I have been to three parties this year and enjoyed them all!"). In this study, specific memories (e.g., "I really enjoyed John's birthday party last spring") constituted task errors. Our main prediction (Hypothesis 1) was that higher AMS scores on the AMT-R would be related to increased depressed mood.

In this study we also included the Operation Span Task (OSPAN; Turner \& Engle, 1989)—a widely used measure of controlled attention. We therefore had two additional hypotheses. First, we hypothesized that lower scores on the OSPAN would be associated with greater AMS on the AMT-R, even with BDI scores partialed out (Hypothesis 2). This therefore provides a reversed version of those analyses presented in Studies 1-4, which sought to show a positive relationship between better performance on executive control tasks and level of AMS on the standard AMT, with depression partialed out. Second, we hypothesized that higher scores on the OSPAN would be associated with lower BDI scores (Hypothesis 3). Because, to our knowledge, there are no published studies investigating OSPAN performance in individuals with depressed mood, we first performed a pilot study with groups of dysphoric and nondysphoric participants to validate the task prior to using it in the present AMT manipulation study. The details of this pilot study are reported at the end of the Method section below.

\section{Method}

\section{Participants}

Thirty-two participants ( 23 women, 9 men) were recruited from the CBU volunteer panel, with a range of BDI scores $(0-36 ; n=15$ in the mild range, mean age $=42.13$ years, $S D=13.51$ years, 10 women, 5 men; $n=$ 11 in the mild-to-moderate range, mean age $=41.18$ years, $S D=12.89$ years, 9 women, 2 men; $n=6$ in the severe range, mean age $=50.17$ years, $S D=9.68$ years, 4 women, 2 men; Shaw et al., 1985). We recruited a higher sample size than in the previous studies of depressed mood (Studies 5-7) because of our concern that the absolute sizes of the critical correlations pertaining to Hypotheses 1 and 2 may be smaller than in the earlier studies (e.g., Study 5) for the reasons outlined in the introduction to the present study. The mean age of participants was 43.3 years $(S D=12.8$ years).

\section{Materials, Measures, and Procedure}

$A M T-R$. In the AMT-R, participants had to avoid responding with specific memories and instead had to generate categorical memories (i.e., memories that were summaries of more than one specific event; see the description of the AMT in Study 1). The instructions for the AMT-R consequently emphasized to participants that

The memory you recall should be of a certain category of event; in other words, a series of similar events that happened to you at different times. So, if I said the word "good," it would not be okay to say "I had a good time at Jane's party," because that does not refer to a category of events. But it would be okay to say "I always enjoy the parties at my friend Jane's house" because that refers to a category of events.

Twelve positive and 12 negative words were selected from Brittlebank et al.'s (1993) study. At the end of the AMT-R, we asked participants to recall the instructions, to ensure that they had understood the task and had remembered the instruction to try to be categoric.

The OSPAN (Turner \& Engle, 1989). In the OSPAN, participants are shown a series of neutral words on cards, for $2 \mathrm{~s}$ each, and asked to remember them. After the final word has been displayed, a simple numeric equation (e.g., $(9 / 3)-2=1$ ) is shown to participants for 4 s. Participants are then required to state whether the answer to the equation is correct or incorrect and, subsequently, to recall the words. The OSPAN consists of 12 trials, 3 using two words, 3 using three words, 3 using four words, and 3 using five words, before each equation. Scores are the total number of words recalled (out of 42). Participants who answered fewer than $85 \%$ of the mathematical questions successfully have their data set aside. This is the partial credit load scoring method as recommended by A. R. A. Conway et al. (2005).

As noted, to our knowledge, there are no published reports of using the OSPAN with individuals in a depressed mood. Therefore, to validate the task for the present study we carried out a pilot study comparing OSPAN performance across a dysphoric group (BDI scores $>15$; Shaw et al., $1985 ; n=20,6$ men, 14 women, mean age $=42.95$ years, $S D=12.53$ years) and a nondysphoric group (BDI scores < 10; Shaw et al., 1985; $n$ $=23,7$ men, 16 women, mean age $=41.83$ years, $S D=11.64$ years $)$, comparable on age, gender ratio, and education level $(p s>.4)$. The results showed that the OSPAN scores for the participants in the dysphoric group $(M=26.85, S D=6.71)$ were significantly lower than for the nondysphoric control participants $(M=31.83, S D=7.80), t(41)=2.23, p<.04, d=$ 0.71 , suggesting that OSPAN performance can vary as a function of dysphoric mood across education-comparable samples, thus providing sufficient validation for the use of the measure in the present study. The AMT-R was administered first, followed by the OSPAN, and finally the BDI.

\section{Results and Discussion}

All participants were able to correctly recall the AMT-R instructions at the end of the task. Unsurprisingly, AMS scores on the AMT-R were low and were not normally distributed $(M=$ 2.94, $S D=3.73)$, and indeed participants generated far more categoric memories $(M=18.53, S D=4.20)$ than specific memories on the AMT-R. A fourth root transformation (Howell, 1997) allowed the AMS data to conform to the requirements for parametric statistics. However, where appropriate, relevant analyses were also performed using nonparametric tests as a cross-check for the results of the parametric analyses using the transformed data.

In line with our first hypothesis, BDI scores $(M=13.56, S D=$ $10.90)$ were correlated positively with AMS (fourth root transformed), $r(30)=.35, p<.05$. This relationship was also signif- 
icant using nonparametric testing with the untransformed data, $r_{\mathrm{s}}(30)=.35, p<.05$. In line with our second hypothesis, performance on the OSPAN $(M=33.91, S D=5.73$; with no participants' data set aside) correlated negatively with AMS (fourth root transformed) even after controlling for BDI scores, $\operatorname{pr}(29)=-.49$, $p<.005$. Finally, in line with our third hypothesis, scores on the OSPAN were negatively associated with BDI scores, $r(30)=$ $-.41, p<.02$.

Taken together these results indicate that the direction of the association between levels of depressed mood and AMS can be reversed by asking participants to generate general (categoric) rather than specific memories on the AMT-R, such that higher levels of depression were associated with greater AMS. These data are again consistent with the predictions of our conceptualization of the AMT as a task significantly dependent on executive control, as outlined in the introduction to this study. This was further confirmed by the fact that performance on the OSPAN was negatively correlated with levels of AMS. The data from Study 8 also replicated the finding from our pilot study (see the Method section of this study) that higher scores on the BDI are associated with lower scores on the OSPAN, this time using a correlational design.

\section{General Discussion}

\section{The Research Rationale and Summary of the Main Findings}

There are a host of research studies indicating that AMS is relatively reduced in individuals with depressed mood, clinical depression, and a variety of other clinical and subclinical states (see Williams et al., in press, for a review). The rationale for the present series of eight studies was to examine systematically, for the first time, the contribution of executive control processes to this widespread finding. In particular, we wanted to examine whether differences in executive control could significantly account for the relationship between depressed mood and reduced AMS.

To this end the eight studies generated five key sets of findings:

1 . Increased depressed mood was significantly related to reduced AMS in six studies (Studies 1, 2, 3, 5, 6, and 7), replicating the established finding in the literature (e.g., Ramponi et al., 2004).

2. Increased depressed mood was also found to be associated with decreased executive control in stratified depressed samples using a standard executive control measure-the OSPAN-using both a group and a correlational design (Study 8).

3. Reduced AMS was associated with poorer performance on tasks that are associated with executive control, including measures of verbal fluency (Study 1), of block design (Study 2), and of fluid intelligence (Study 4), all independent of depressed mood. Reduced AMS also related to numbers of errors on verbal fluency, design fluency, and the Alternate Uses Test (all three summed; Study 2), on the Porteus Maze Task (Study 2), and on a numbergeneration task (Study 3), independent of depressed mood. A reversed version of the AMT (the AMT-R) revealed that AMS was associated in the expected way with operation span (on the OSPAN; see Study 8), again independent of depressed mood. These different relationships were demonstrated variously in unselected healthy participants, in participants with subclinical depressed mood, and in a clinical group of participants with eating disorders.
4. Executive control (in the form of verbal fluency) was found to mediate the relationship between both depressed mood and a diagnosis of eating disorder, as predictors, and AMS, as the criterion (Study 1).

5. The magnitude and direction of the relationship between depressed mood and AMS was altered by experimentally manipulating the task parameters of the AMT so as to either increase or decrease the demands on executive control. Manipulating the content of the AMT cue words (Study 6), the addition of mental load (Study 7), and reversal of the AMT instructions (Study 8), in participant samples with a range of depressed mood scores, all served to change the relationship between depressed mood and AMS in ways that one would predict if this relationship was driven to a significant extent by a reduction in executive control capabilities associated with depressed mood.

Taken together these five sets of findings provide support for the view that the oft-replicated relationship between reduced AMS and various clinical and subclinical states (see Williams et al., in press, for a review) can be significantly accounted for by reduced executive control in those states. This is potentially important for a number of reasons. First, it provides a new and different way of thinking about AMS effects, which are at present incompletely understood. Second, because executive control provides a potential account of AMS effects that is not centered on autobiographical memory processes (unlike, for instance, the affect regulation hypothesis outlined in the introduction), it permits a closer integration of the AMT literature with the literature on other aspects of cognitive processing in depression (as well as in other clinical and subclinical states; e.g., Brewin \& Beaton, 2004). This facilitates the development of broad, integrated cognitive theories of depression, as opposed to specific, bespoke theories that focus on discrete cognitive phenomena. Finally, an executive control analysis of AMS effects establishes a bridge between important aspects of the mainstream cognitive psychology literature and phenomena in abnormal psychology. Such bridges are clearly going to be important in efforts to develop macrotheoretical models of cognitionemotion relations (see Dalgleish, 2004, for a discussion).

In the remainder of this section we seek to do three things. First, we discuss the nature of any executive control deficits in depression and how they relate to reduced AMS. Second, we revisit the key findings in the AMS literature and explore how they might be explained in times of reduced executive control. Finally, we consider limitations and future directions for this research endeavor.

\section{The Nature of the Executive Control Explanation for AMS}

Throughout the article we have conceptualized individual differences in executive control as differences in the ability to exert controlled attention in the pursuit of (task) goals, particularly in the face of automatically generated distracter material (Barrett et al., 2004; Rosen \& Engle, 1997; see the Results and Discussion section of Study 2). However, this broad definition is likely to encompass a number of different aspects of cognitive functioning (Engle et al., 1999; Hertel, 2000), some or all of which may be critical in understanding AMS effects. Although the present data clearly indicate for the first time a role for executive control in accounting for AMS effects, they cannot provide definitive insight into exactly which of these different facets of executive control are 
most important. In this vein, a number of candidates present themselves.

First, AMS may be a function of difficulties with inhibition of distracters (Engle, Conway, Tuholski, \& Shisler, 1995). As discussed in Footnote 1, there is a healthy debate within cognitive psychology about the extent to which differential processing of certain information at the expense of distracting information is either a function of direct (i.e., strong) inhibition of the latter or is simply a function of the former receiving preferential processing (i.e., weak inhibition; see, e.g., Anderson \& Spellman, 1995; Dalgleish et al., 1999; MacLeod et al., 2003). This debate notwithstanding, it is plausible that depressed mood is associated with deficits in either strong or weak inhibition with respect to distracting information in the context of tasks requiring the application of controlled attention, and there are data consistent with deficits in inhibition associated with depression on such tasks (e.g., Power, Dalgleish, \& Claudio, 2000; see Mathews \& MacLeod, 2005, for some discussion).

Second, the AMS effects reported here may reflect a difficulty in maintaining the entire set of task goals in active memory in the face of distracting information (Engle et al., 1999). For example, on the AMT the principal goal of retrieving autobiographical material may remain in focus, but the subgoal that the material has to be specific in nature may be neglected. This does not mean to imply either that participants are unaware of the goals or that they would have forgotten them; rather, the argument is that executive control is not applied in a way that serves to hold in memory all of the goals and furthermore to check all candidate responses against each goal for every trial to prevent errors. This aspect of executive control has perhaps best been elucidated in the literature on goal neglect (Duncan et al., 1996; see Engle et al., 1999, for a discussion).

A third possibility is that those individuals with seemingly impaired executive control utilize a different balance of automatic and controlled processing (e.g., Barrett et al., 2004). This may not reflect a lack of motivation or initiative to perform complex tasks like the AMT. Instead, it may reflect previous learning that attempts at controlled processing are unsuccessful and therefore not worth the investment of limited attentional resources. In effect, individuals who appear impaired in executive control may adopt an automatic processing mode as a way to optimize their performance on cognitively demanding tasks. There is some indication that individuals characterized by different levels of executive control differ in their use of automatic and controlled processing strategies during executive-control-demanding tasks. For example, in Rosen and Engle's (1997) study described above, individuals lower in executive control (or working memory capacity, in their terms) relied on automatic spreading activation for retrieval of category exemplars in a fluency task, whereas those higher in working memory capacity used controlled attention to guide their search after a certain point in time. As a result, those lower in executive control in this study might be best described by the cognitive miser metaphor (Fiske \& Taylor, 1991). Cognitive misers are seen as having severely limited executive control capabilities and as a result adopt strategies that simplify the need for controlled attention. Although they may have an array of goals or motives, they do not have the executive control capability to maintain goal-relevant processing in the face of complex situa- tions, such that they end up emphasizing efficiency over any other processing goal.

Related to these questions about the exact nature of any cognitive control deficits in depression is the issue of why depressed mood should lead to problems with executive control in the first place. Perhaps the strongest candidate explanation is that the ruminative processes and task-irrelevant thoughts that are associated with depression interfere with the efficient and effective use of controlled attention in the performance of tasks such as the AMT in the ways outlined above. Of note here are Watkins and Teasdale's (2001) data showing that experimental manipulations to increase rumination serve to reduce AMS in depressed participants. It would be interesting to examine whether these sorts of effects were mediated by shifts in executive control capability.

\section{An Executive Control Account of the Key Findings From the AMS Literature}

It is as yet unclear to what extent individual differences in executive control can explain all of the data on AMS effects in the literature (see the introduction to this article), as the relevant studies have yet to be carried out. However, given that executive control appears to be a significant factor in reduced AMS, one should be able to generate a plausible account of the key findings in this literature in terms of such control. In this section therefore we examine the potential explanatory breadth of individual differences in executive control, beginning with the relationship between AMS and trauma.

An executive control analysis of the widely replicated associations between reduced AMS and both trauma history and symptoms of posttraumatic stress (e.g., Hermans et al., 2004; Kuyken \& Brewin, 1995) would propose that the experience of trauma and symptoms reflecting attempts to process that trauma, including intrusive cognitions and affect (see Dalgleish, 2004), compromise executive control. Consistent with this, there is a body of research showing that traumatized individuals with significant posttraumatic stress are impaired on a range of executive control tasks (see Knight \& Taft, 2004, for a review). Similarly, in a series of experiments Ellis, Moore, Varner, Ottaway, and Becker (1997) showed that the presence of irrelevant thoughts predicts poorer executive control task performance in individuals experiencing affective states.

A similar analysis can be offered for other aspects of the reduced-AMS literature: in particular, why reduced AMS (a) indexes a relatively poor prognosis for clinical outcome in longitudinal studies in different clinical groups (e.g., Brittlebank et al., 1993), (b) is associated with impoverished social problem solving (e.g., Goddard et al., 1996; Goddard, Dritschel, \& Burton, 1997), and (c) relates to participants' difficulty being specific and fluent about the future (Williams et al., 1996; see also Study 1 of the present article). The thesis here would be that reduced executive control to a significant extent drives both poor problem solving (e.g., Kyllonen \& Christal, 1990, reported correlations of .8-.9 between executive control and reasoning ability in a series of studies with over 2,000 participants) and low specificity about the future (see Study 1), as well as reduced AMS. It is interesting to note that when AMS is experimentally manipulated, it significantly affects future event specificity (Williams et al., 1996) and problem-solving ability (Williams et al., 2006, Studies 4 and 5), 
thus suggesting that level of AMS may itself have a causal link with these variables. What is unclear at present is the extent to which this causal relationship may be mediated by executive control. Finally, relative difficulties with problem solving, being specific about the future, and dealing with intrusive cognitions and affect (as discussed above) are likely to ensure that individuals with lower executive control will recover less well from clinical states in longitudinal studies.

The relationship between low executive control and reduced AMS in individuals recovered from depression (e.g., Mackinger, Pachinger, et al., 2000) could reflect two underlying cognitive processes. First, individuals with lower levels of executive control may be more vulnerable to depression in the first place (i.e., executive control is a risk factor for the onset of depression), for the same reasons that lower executive control is associated with poor recovery from depression. There is some research consistent with this; for example, Klein and Boals (2001) showed that individuals low in executive control (working memory capacity) reacted with greater affect to stressful life events relative to individuals high in executive control.

Second, there is a wealth of evidence that individuals recovered from depression chronically need to apply executive control to suppress, block, or gate negative self-related information (see Wenzlaff \& Wegner, 2000, for a review). So, for example, Wenzlaff and Bates (1998) showed that individuals vulnerable to depression exhibited certain depressogenic self-referent cognitive biases but only when placed under conditions of mental load. Under conditions of no load they were indistinguishable from never-depressed control participants. It may therefore be the case that executive control capabilities are chronically compromised in depression-vulnerable individuals in the service of dealing with negative self-referent material. There would therefore be fewer such "resources" available for executive-control-demanding tasks such as the AMT.

It is interesting to note that on the basis of these arguments one would also argue that impoverished executive control would be a risk factor for the onset of traumatic stress disorders following trauma exposure due to associated difficulties in dealing with automatically generated distracting information such as intrusive thoughts, images, and nightmares about the trauma. In line with this possibility we know that lower scores on standard intelligence tests are a risk factor for the development of posttraumatic stress states in trauma-exposed samples (see Brewin, Andrews, \& Valentine, 2000; Ozer, Best, Lipsey, \& Weiss, 2003), and this may reflect the fact that individuals relatively low in executive control (who would tend to score lower on general IQ measures) are at greater risk. A related issue has been examined experimentally by Brewin and Beaton (2004). They showed that higher numbers of unwanted intrusive thoughts on the White Bear Suppression Task (Wegner \& Zanakos, 1994) were associated with diminished executive control on the OSPAN in healthy participants.

\section{Other Factors Involved in AMS}

The causal link between executive control and AMT performance that we have demonstrated here does not of course rule out the possibility that other psychological processes might also influence AMS (see Williams et al., in press). For example, we have argued elsewhere that a key factor in understanding reduced AMS in various clinical groups may be the fact that the AMT cue words map onto particular pathological generic sets of concerns or beliefs (schemas) of the participants (Dalgleish et al., 2003), which may themselves be chronically primed and reinforced by ruminative processes (Watkins \& Teasdale, 2001). The proposal is that in such instances a given cue word is likely to activate or bring to mind a candidate generic response reflecting these negative schemas. As only specific memories count as correct responses on the standard AMT, these generic candidates need to be inhibited as responses in the service of successful task performance. This analysis suggests that individuals suffering from clinical conditions characterized by such underlying negative schematic representations will have a relatively greater number of generic candidate responses to inhibit compared with healthy control participants. It is easy to see how this relative abundance of to-be-inhibited generic responses could interact with impoverished executive control in clinical participants to bring about a markedly reduced AMS effect in such groups.

Another potential explanatory variable for reduced AMS effects is of course the affect regulation hypothesis outlined in the introduction. The present findings considered together (especially those from Study 8) do not support an affect regulation view of the relationship between depressed mood and AMS. However, it may be the case that a different pattern of results would emerge in a significantly traumatized sample. Indeed, findings from Study 1, in which we did not find a relationship between verbal fluency and self-reported parental abuse to mirror the established relationship between AMS and this variable (Dalgleish et al., 2003), suggest that a history of trauma and/or posttraumatic symptomatology may exert effects on AMS somewhat independently of any effects of executive control, though this remains an empirical question.

\section{Conclusions, Limitations, and Future Directions}

The present series of studies has shown clearly for the first time that individual differences in executive control play a key role in driving performance on the AMT, indicating that these differences are an important element in accounting for the widely reported relationship between subclinical and clinical states and reduced AMS on this task (Williams et al., in press). One limitation of the present studies is that they focused predominantly on reduced AMS in subclinical samples (though see Study 1). Further elucidation of executive control effects on AMS in different clinical groups therefore awaits additional empirical work. Second, we have concentrated on the relationships between AMS and depressed mood, and with the exception of Study 1, we have neglected the relationship between AMS and various indices of trauma.

In line with these comments, future research directions should include examining the executive control hypothesis of AMT performance in a wider range of clinical and clinically vulnerable groups, in longitudinal studies, and in populations with experience of trauma. Further work is also needed to investigate exactly which aspects of subclinical and clinical states lead to impairments in executive control and how these aspects can be addressed through clinical intervention. Another key focus for future research is to elucidate exactly which aspects of executive control are implicated in the reduced AMS effect and, more generally, which aspects of executive control are compromised in depressed mood and other 
subclinical and clinical states. Related to this, the door is now open to examine in theoretically motivated ways the relationship between reduced AMS and other aspects of cognitive task performance in depression and other clinical and subclinical states (e.g., Brewin \& Beaton, 2004).

\section{References}

American Psychiatric Association. (1994). Diagnostic and statistical manual of mental disorders (4th ed.). Washington, DC: Author.

Anderson, M. C., \& Spellman, B. A. (1995). On the status of inhibitory mechanisms in cognition: Memory retrieval as a model case. Psychological Review, 102, 68-100.

Au Yeung, C., Dalgleish, T., Golden, A. M., \& Schartau, P. (2006). Reduced specificity of autobiographical memories following a negative mood induction. Behaviour Research and Therapy, 44, 1481-1490.

Baddeley, A. D. (1983). Working memory. Philosophical Transactions of the Royal Society of London, Series B, 302, 311-324.

Baddeley, A. (1986). Working memory. Oxford, England: Oxford University Press.

Baddeley, A. D., Emslie, H., \& Nimmo-Smith, I. (1992). The Speed and Capacity of Language Processing (SCOLP) Test. Bury St. Edmunds, Suffolk, England: Thames Valley Test Company.

Barnard, P. J., Watkins, E. R., \& Ramponi, C. (2006). Reducing specificity of autobiographical memory in non-clinical participants: The role of rumination and schematic models. Cognition \& Emotion, 20, 328-350.

Barrett, L. F., Tugade, M. M., \& Engle, R. W. (2004). Individual differences in working memory capacity and dual process theories of the mind. Psychological Bulletin, 130, 553-573.

Beck, A. T., Steer, R. A., \& Garbin, M. G. (1988). Psychometric properties of the Beck Depression Inventory: Twenty-five years of evaluation. Clinical Psychology Review, 8, 77-100.

Beck, A. T., Ward, C. H., Mendelson, M., Mock, J., \& Erbaugh, J. (1961). An inventory for measuring depression. Archives of General Psychiatry, 4, 53-63.

Boudewyn, A. C., \& Liem, J. H. (1995). Childhood sexual abuse as a precursor to depression and self-destructive behavior in adulthood. Journal of Traumatic Stress, 8, 445-449.

Brewin, C. R., Andrews, B., \& Valentine, J. D. (2000). Meta-analysis of risk factors for posttraumatic stress disorder in trauma-exposed adults. Journal of Consulting and Clinical Psychology, 68(5), 748-766.

Brewin, C. R., \& Beaton, A. (2004). Thought suppression, intelligence, and working memory capacity. Behaviour Research and Therapy, 40, 923930.

Brewin, C. R., Reynolds, M., \& Tata, P. (1999). Autobiographical memory processes and the course of depression. Journal of Abnormal Psychology, 108(3), 511-517.

Brittlebank, A. D., Scott, J., Williams, J. M. G., \& Ferrier, I. N. (1993). Autobiographical memory in depression: State or trait marker? British Journal of Psychiatry, 162, 118-121.

Burt, D., Zembar, M. J., \& Niederehe, G. (1995). Depression and memory impairment: A meta-analysis of the association, its pattern, and specificity. Psychological Bulletin, 117, 285-305.

Cattell, R. B., \& Cattell, A. (1960). Test of " $g$ ": Culture fair. Champaign, IL: Institute of Personality and Ability Testing.

Cohen, J. D. (1988). Statistical power analyses for the behavioral sciences (2nd ed.). New York: Academic Press.

Conway, A. R. A., Kane, M. J., Bunting, M. F., Hambrick, D. Z., Wilhelm, O., \& Engle, R. W. (2005). Working memory span tasks: A methodological review and user's guide. Psychonomic Bulletin \& Review, 12, $769-786$.

Conway, A. R. A., Kane, M. J., \& Engle, R. W. (2003). Working memory capacity and its relation to general intelligence. Trends in Cognitive Sciences, 7, 547-552.
Conway, M. A., \& Pleydell-Pearce, C. W. (2000). The construction of autobiographical memories in the self-memory system. Psychological Review, 107, 261-288.

Corey, D. M., Dunlap, W. P., \& Burke, M. J. (1998). Averaging correlations: Expected values and bias in combined Pearson $r$ s and Fisher's $z$ transformations. Journal of General Psychology, 125, 246-261.

Cronbach, L., \& Meehl, P. (1955). Construct validity in psychological tests. Psychological Bulletin, 52, 281-302.

Dagenbach, D., \& Carr, T. H. (Eds.). (1994). Inhibitory processes in attention memory and language. San Diego, CA: Academic Press.

Dalgleish, T. (2004). Cognitive approaches to posttraumatic stress disorder: The evolution of multirepresentational theorizing. Psychological Bulletin, 130, 228-260.

Dalgleish, T., Mathews, A., \& Wood, J. (1999). Inhibition processes in cognition and emotion: A special case? In T. Dalgleish \& M. Power (Eds.), Handbook of cognition and emotion (pp. 243-266). Chichester, England: Wiley.

Dalgleish, T., Spinks, H., Yiend, J., \& Kuyken, W. (2001). Autobiographical memory style in seasonal affective disorder and its relationship to future symptom remission. Journal of Abnormal Psychology, 110, 335340

Dalgleish, T., Tchanturia, K., Serpell, L., Hems, S., De Silva, P., \& Treasure, J. (2003). Self-reported parental abuse relates to autobiographical memory style in patients with eating disorders. Emotion, 3, 211222.

de Decker, A., Hermans, D., Raes, F., \& Eelen, P. (2003). Autobiographical memory specificity and trauma in inpatient adolescents. Journal of Clinical Child and Adolescent Psychology, 32, 22-31.

Duncan, J., Emslie, H., \& Williams, P. (1996). Intelligence and the frontal lobe: The organization of goal directed behavior. Cognitive Psychology, $30,257-303$.

Ellis, H. C., \& Ashbrook, P. W. (1988). Resource allocation model of the effects of depressed mood states on memory. In J. Forgas (Ed.), Affect, cognition, and social behaviour (pp. 25-43). Toronto, Ontario, Canada: Hogrefe.

Ellis, H. C., Moore, B. A., Varner, L. J., Ottaway, S. A., \& Becker, A. S. (1997). Depressed mood, task organization, cognitive interference, and memory: Irrelevant thoughts predict recall performance. Journal of Social Behavior and Personality, 12, 453-470.

Engle, R. W. (2002). Working memory capacity as executive attention. Current Directions in Psychological Science, 11, 19-23.

Engle, R. W., Conway, A. R. A., Tuholski, S. W., \& Shisler, R. J. (1995). A resource account of inhibition. Psychological Science, 6, 122-125.

Engle, R. W., \& Kane, M. J. (2004). Executive attention, working memory capacity, and a two-factor theory of cognitive control. The Psychology of Learning and Motivation: Advances in Research and Theory, 44, 145199.

Engle, R. W., Tuholski, S. W., Laughlin, J. E., \& Conway, A. R. A. (1999). Working memory, short-term memory, and general fluid intelligence: A latent variable approach. Journal of Experimental Psychology: General, 128, 309-331.

Fiske, S. T., \& Taylor, S. E. (1991). Social cognition. New York: McGrawHill.

Goddard, L., Dritschel, B., \& Burton, A. (1996). The role of autobiographical memory in social problem-solving and depression. Journal of $A b$ normal Psychology, 105, 609-616.

Goddard, L., Dritschel, B., \& Burton, A. (1997). Social problem solving and autobiographical memory in non-clinical depression. British Journal of Clinical Psychology, 36, 449-451.

Godley, J., Tchanturia, K., MacLeod, A., \& Schmidt, U. (2001). Futuredirected thinking in eating disorders. British Journal of Clinical Psychology, 40, 281-295.

Hartlage, S., Alloy, L. B., Vazquez, C., \& Dykman, B. (1993). Automatic 
and effortful processing in depression. Psychological Bulletin, 113, 247-278.

Harvey, A. G., Bryant, R. A., \& Dang, S. T. (1998). Autobiographical memory in acute stress disorder. Journal of Consulting and Clinical Psychology, 66, 500-506.

Hasher, L., \& Zacks, R. T. (1979). Automatic and effortful processes in memory. Journal of Experimental Psychology: General, 108, 356-388.

Henderson, D., Hargreaves, I., Gregory, S., \& Williams, J. M. G. (2002). Autobiographical memory and emotion in a non-clinical sample of women with and without a reported history of childhood sexual abuse. British Journal of Clinical Psychology, 41, 129-142.

Hermans, D., Van den Broeck, K., Belis, G., Raes, F., Pieters, G., \& Eelen, P. (2004). Trauma and autobiographical memory specificity in depressed inpatients. Behavior Research and Therapy, 42, 775-789.

Hertel, P. T. (2000). The cognitive-initiative account of depression-related impairments in memory. In D. Medin (Ed.), The psychology of learning and motivation (Vol. 39, pp. 47-71). New York: Academic Press.

Hertel, P. T., \& Hardin, T. S. (1990). Remembering with and without awareness in a depressed mood: Evidence of deficits in initiative. Journal of Experimental Psychology: General, 119, 45-59.

Hertel, P. T., \& Rude, S. (1991). Depressive deficits in memory: Focusing attention improves subsequent recall. Journal of Experimental Psychology: General, 120, 301-309.

Hipwell, A. E., Reynolds, S., \& Pitts Crick, E. (2004). Cognitive vulnerability to postnatal depressive symptomatology. Journal of Reproductive and Infant Psychology, 22, 211-227.

Horn, J. L., \& Cattell, R. B. (1967). Age differences in fluid and crystallized intelligence. Acta Psychologica, 26, 107-129.

Howell, D. C. (1997). Statistical methods for psychology (4th ed.). Belmont, CA: Duxbury Press.

Jones-Gotman, M., \& Milner, B. (1977). Design fluency: The invention of nonsense drawings after focal cortical lesions. Neuropsychologia, 15, 653-674.

Kane, M. J., \& Engle, R. W. (2002). The role of prefrontal cortex in working-memory capacity, executive attention and general fluid intelligence: An individual differences perspective. Psychonomic Bulletin \& Review, 9, 637-671.

Kane, M. J., \& Engle, R. W. (2003). Working memory capacity and the control of attention: The contributions of goal neglect, response competition, and task set to Stroop interference. Journal of Experimental Psychology: General, 132, 47-70.

Kangas, M., Henry, J. L., \& Bryant, R. A. (2005). A prospective study of autobiographical memory and posttraumatic stress disorder following cancer. Journal of Consulting and Clinical Psychology, 73, 293-299.

Klein, K., \& Boals, A. (2001). The relationship of life-event stress and working memory capacity. Applied Cognitive Psychology, 15, 565-579.

Knight, J. A., \& Taft, C. T. (2004). Assessing neuropsychological concomitants of trauma and PTSD. In T. M. Keane \& J. P. Wilson (Eds.), Assessing psychological trauma and PTSD (pp. 344-388). New York: Guilford Press.

Kučera, H., \& Francis, W. N. (1967). Computational analysis of presentday American English. Providence, RI: Brown University Press.

Kuyken, W., \& Brewin, C. R. (1995). Autobiographical memory functioning in depression and reports of early abuse. Journal of Abnormal Psychology, 104, 585-591.

Kuyken, W., \& Dalgleish, T. (1995). Autobiographical memory and depression. British Journal of Clinical Psychology, 33, 89-92.

Kuyken, W., Howell, R., \& Dalgleish, T. (2006). Overgeneral autobiographical memory in depressed adolescents with, versus without, a reported history of trauma. Journal of Abnormal Psychology, 115, 387396.

Kyllonen, P. C., \& Christal, R. E. (1990). Reasoning ability is (little more than) working memory capacity?! Intelligence, 14, 389-433.
Lezak, M. D. (1995). Neuropsychological assessment (3rd ed.). New York: Oxford University Press.

Mackinger, H. F., Loschin, G. G., \& Leibetseder, M. M. (2000). Prediction of postnatal affective changes by autobiographical memories. European Psychologist, 5, 52-61.

Mackinger, H. F., Pachinger, M. M., Leibetseder, M. M., \& Fartacek, R. R. (2000). Autobiographical memories in women remitted from major depression. Journal of Abnormal Psychology, 109, 331-334.

MacKinnon, D. P., Lockwood, C. M., Hoffman, J. M., West, S. G., \& Sheets, V. (2002). A comparison of methods to test mediation and other intervening variable effects. Psychological Methods, 7, 83-104.

MacLeod, C. M., Dodd, M. D., Sheard, E. D., Wilson, D. E., \& Bibi, U. (2003). In opposition to inhibition. The Psychology of Learning and Motivation, 43, 163-214.

Mathews, A., \& MacLeod, C. (2005). Cognitive vulnerability to emotional disorders. Annual Review of Clinical Psychology, 1, 167-195.

McNally, R. J., Litz, B. T., Prassas, A., Shin, L. N., \& Weathers, F. (1994). Emotional priming and autobiographical memory in posttraumatic stress disorder. Cognition \& Emotion, 8, 351-368.

Miyake, A., \& Shah, P. (Eds.). (1999). Models of working memory: Mechanisms of active maintenance and executive control. Cambridge, England: Cambridge University Press.

Norman, D. A., \& Shallice, T. (1986). Attention to action: Willed and automatic control of behaviour. In R. J. Davidson, G. E. Schwartz, \& D. Shapiro (Eds.), The design of everyday things (pp. 1-18). New York: Doubleday.

Ozer, E. J., Best, S. R., Lipsey, T. L., \& Weiss, D. S. (2003). Predictors of posttraumatic stress disorder and symptoms in adults: A meta-analysis. Psychological Bulletin, 129, 52-73.

Park, R. J., Goodyer, I. M., \& Teasdale, J. (2002). Categoric overgeneral autobiographical memory in adolescents with major depressive disorder. Psychological Medicine, 32, 267-276.

Parker, G., Roussos, J., Hadzi-Pavlovic, D., Mitchell, P., Wilhelm, K., \& Austin, M.-P. (1997). The development of a refined measure of dysfunctional parenting and assessment of its relevance in patients with affective disorders. Psychological Medicine, 27, 1193-1203.

Porteus, S. D. (1965). Porteus Maze Test: Fifty years' application. Palo Alto, CA: Pacific Books.

Posner, M. I., \& Snyder, C. R. R. (1975). Facilitation and inhibition in the processing of signals. In S. Dornic (Ed.), Attention and performance $V$ (pp. 669-682). London: Academic Press.

Power, M. J., Dalgleish, T., \& Claudio, V. (2000). The directed forgetting task: Application to emotionally valent material. Journal of Affective Disorders, 57, 147-157.

Preacher, K. J., \& Hayes, A. F. (2004). SPSS and SAS procedures for estimating indirect effects in simple mediation models. Behavior Research Methods, Instruments, \& Computers, 36, 717-731.

Ramponi, C., Barnard, P., \& Nimmo-Smith, I. (2004). Recollection deficits in dysphoric mood: An effect of schematic models and executive mode? Memory, 12, 655-670.

Rosen, V. M., \& Engle, R. W. (1997). The role of working memory capacity in retrieval. Journal of Experimental Psychology: General, 126, 211-227.

Schneider, W., \& Shiffrin, R. M. (1977). Controlled and automatic human information processing: I. Detection, search, and attention. Psychological Review, 84, 1-66.

Scott, S. K., Barnard, P. J., \& May, J. (2001a). Specifying executive representations and processes in number generation tasks. Quarterly Journal of Experimental Psychology: Human Experimental Psychology, 54(A), 641-664.

Scott, S. K., Barnard, P. J., \& May, J. (2001b). When the central executive lets us down: Schemas, attention, and load in a generative working memory task. Memory, 9, 209-221.

Shaw, B. F., Vallis, T. M., \& McCabe, S. B. (1985). The assessment of the 
severity and symptom patterns in depression. In E. E. Beckham \& W. R. Leber (Eds.), Handbook of depression: Treatment, assessment and research (pp. 372-407). Homewood, IL: Dorsey Press.

Startup, M., Heard, H., Swales, M., Jones, B., Williams, J. M. G., \& Jones, R. S. P. (2001). Autobiographical memory and parasuicide in borderline personality disorder. British Journal of Clinical Psychology, 40, 113120.

Turner, M. L., \& Engle, R. W. (1989). Is working memory capacity task dependent? Journal of Memory and Language, 28(2), 127-154.

Unsworth, N., \& Engle, R. W. (2005). Working memory capacity and fluid abilities: Examining the correlation between Operation Span and Ravens. Intelligence, 33, 67-81.

van Vreeswijk, M. F., \& de Wilde, E. J. (2004). Autobiographical memory specificity, psychopathology, depressed mood and the use of the Autobiographical Memory Test: A meta-analysis. Behaviour Research and Therapy, 42, 731-743.

Watkins, E., \& Teasdale, J. (2001). Rumination and overgeneral memory in depression: Effects of self-focus and analytic thinking. Journal of Abnormal Psychology, 110, 353-357.

Wechsler, D. (1998). WAIS-III administration and scoring manual. London: Psychological Corporation.

Wegner, D. M., \& Zanakos, S. (1994). Chronic thought suppression. Journal of Personality, 62, 615-640.

Wenzlaff, R. M., \& Bates, D. E. (1998). Unmasking a cognitive vulnerability to depression: How lapses in mental control reveal depressive thinking. Journal of Personality and Social Psychology, 75(6), 15591571.

Wenzlaff, R. M., \& Wegner, D. M. (2000). Thought suppression. In S. T. Fiske (Ed.), Annual review of psychology (Vol. 51, pp. 59-91). Palo Alto, CA: Annual Reviews.

Wessel, I., Meeren, M., Peeters, F., Arntz, A., \& Merckelbach, H. (2001). Correlates of autobiographical memory specificity: The role of depression, anxiety and childhood trauma. Behaviour Research and Therapy, 39, 409-421.

Williams, J. M. G. (1992). The psychological treatment of depression. London: Routledge.

Williams, J. M. G. (1996). Depression and the specificity of autobiograph- ical memory. In D. C. Rubin (Ed.), Remembering our past: Studies in autobiographical memory (244-267). Cambridge, England: Cambridge University Press.

Williams, J. M. G., Barnhofer, T., Crane, C., Hermans, D., Raes, F., Watkins, E., \& Dalgleish, T. (in press). Autobiographical memory specificity and emotional disorder. Psychological Bulletin.

Williams, J. M. G., \& Broadbent, K. (1986). Autobiographical memory in suicide attempters. Journal of Abnormal Psychology, 95, 144-149.

Williams, J. M. G., Chan, S., Crane, C., Barnhofer, T., Eade, J., \& Healy, H. (2006). Retrieval of autobiographical memories: The mechanisms and consequences of truncated search. Cognition and Emotion, 20, 351-382.

Williams, J. M. G., \& Dritschel, B. H. (1992). Categoric and extended autobiographical memories. In M. Conway, D. Rubin, H. Spinnler, \& W. A. Wagenaar (Eds.), Theoretical perspectives on autobiographical memory (pp. 391-412). Dordrecht, the Netherlands: Kluwer Academic Publishers.

Williams, J. M. G., Ellis, N. C., Tyers, C., MacLeod, A. K., \& Rose, G. (1996). Specificity of autobiographical memory and imageability of the future. Memory \& Cognition, 24, 116-125.

Williams, J. M. G., Healy, H. H., \& Ellis, N. C. (1999). The effect of imageability and predictability of cues in autobiographical memory. Quarterly Journal of Experimental Psychology: Human Experimental Psychology, 52(A), 555-579.

Williams, J. M. G., Stiles, W. B., \& Shapiro, D. (1999). Cognitive mechanisms in the avoidance of painful and dangerous thoughts: Elaborating the assimilation model. Cognitive Therapy and Research, 23, 285-306.

Wilson, M. (1987). MRC psycholinguistic database: Machine usable dictionary (Version 2.00). Didcott, United Kingdom: Informatics Division, Science and Engineering Research Council, Rutherford Appleton Laboratory.

Zigmond, A. S., \& Snaith, R. P. (1983). The Hospital Anxiety and Depression Scale. Acta Psychiatrica Scandinavica, 67, 361-370.

Received August 5, 2005

Revision received June 5, 2006

Accepted June 15, 2006

\section{E-Mail Notification of Your Latest Issue Online!}

Would you like to know when the next issue of your favorite APA journal will be available online? This service is now available to you. Sign up at http://watson.apa.org/ notify/ and you will be notified by e-mail when issues of interest to you become available! 